\title{
Induction of $\mathrm{X}$-chromosome Inactivation by the Histone Demethylase SMCX/KDM5C
}

Srimonta Gayen ${ }^{1}$, Emily Maclary ${ }^{1}$, Yumie Murata-Nakamura ${ }^{1}$, Christina N. Vallianatos ${ }^{1}$, Robert S. Porter ${ }^{1}$, Patricia M. Garay ${ }^{1,2}$, Shigeki Iwase ${ }^{1,2 *}$, and Sundeep Kalantry ${ }^{1 *}$

${ }^{1}$ Department of Human Genetics

2 Neuroscience Graduate Program

University of Michigan Medical School, Ann Arbor, MI 48109-5618.

*Correspondence to: kalantry@umich.edu or siwase@umich.edu

Keywords: X-chromosome inactivation, X-inactivation escapee, histone demethylation. 


\section{SUMMARY}

$X Y$ male and $X X$ female mammals equalize $\mathrm{X}$-linked gene expression through the mitoticallystable transcriptional inactivation of an X-chromosome in females. Although most genes are silent on the inactive-X, some escape silencing and are expressed at higher levels in females vs. males. Here, we show that the escapee $\operatorname{Smcx} / K d m 5 c$, encoding a histone H3K4me2/3 demethylase, underlies the female-specific induction of X-inactivation. Mouse embryonic epiblast cells and differentiating embryonic stem cells (ESCs) lacking SMCX show reduced expression of Xist RNA, which is required for X-inactivation. Smcx-heterozygous epiblast cells do not silence X-linked genes efficiently, despite robust Xist expression. Overexpression of mouse or human SMCX, but not a catalytically-inactive SMCX or the Y-chromosome homolog SMCY, is sufficient to induce Xist and, separately, to silence X-linked genes in male ESCs. Finally, SMCX dose is inversely correlated with H3K4me2 at X-linked loci. Thus, Xinactivation initiates through the evolutionarily conserved, dose-dependent function of the histone demethylase SMCX. 


\section{Main Text}

$\mathrm{X}$-inactivation is an evolutionarily conserved process that equalizes $\mathrm{X}$-linked gene expression between male and female mammals by silencing genes on one of the two Xchromosomes in females $^{1-3}$. Once inactivated, replicated copies of the X-chromosome are maintained as inactive throughout future rounds of cell division ${ }^{3}$, thus making $\mathrm{X}$-inactivation a paradigm of epigenetic inheritance. Stable X-inactivation requires the Xist long noncoding $\mathrm{RNA}^{4,5}$, which is upregulated from and physically coats the future inactive $\mathrm{X}$-chromosome ${ }^{6-10}$. Xist RNA accumulation recruits proteins that in turn are believed to silence X-linked genes ${ }^{11-13}$. However, the precise order of molecular mechanisms by which female cells upregulate Xist RNA and undergo X-inactivation remain obscure.

We hypothesized that the increased dose in $X X$ females compared to $X Y$ males of one or more $\mathrm{X}$-linked genes that escape $\mathrm{X}$-inactivation induces Xist and, separately, silences $\mathrm{X}$-linked genes selectively in females ${ }^{14}$. We thus nominated the X-inactivation escapee $S m c x / K d m 5 c$ as a candidate inducer of Xist and X-linked gene silencing, for the following reasons. First, SMCX demethylates histone $\mathrm{H} 3$ di- and tri- methylated at lysine $4(\mathrm{H} 3 \mathrm{~K} 4 \mathrm{me} 2 \text { and } \mathrm{H} 3 \mathrm{~K} 4 \mathrm{me} 3)^{15,16}$, which are chromatin marks associated with active gene expression ${ }^{17,18}$. Second, hypomethylation of $\mathrm{H} 3 \mathrm{~K} 4$ is associated with Xist induction and silencing of X-linked genes on the inactive- $\mathrm{X}^{19,20}$. Finally, unlike many other escapees, the Smcx gene escapes $\mathrm{X}$-inactivation in both mouse and human ${ }^{21-24}$, suggesting an evolutionarily conserved dose-dependent function.

\section{Smcx is required to induce Xist RNA}

To test for a role of $S m c x$ in X-inactivation, we took advantage of a conditional $S m c x^{\mathrm{fl}}$ allele to generate homozygous $S m c x^{\Delta / \Delta}$ and hemizygous $S m c x^{\Delta / Y}$ mice ${ }^{25}$. Strikingly, although $S m c x^{\Delta / Y}$ males were viable, $S m c x^{\Delta / \Delta}$ females were not (Fig. 1a). We also crossed $S m c x^{\mathrm{fl} / \Delta}$ females 
harboring a Zp3-Cre transgene with $S m c x^{\Delta / Y}$ males. The expression of the Zp3-Cre transgene deletes floxed alleles in growing oocytes and thus ensures that the resulting embryos are depleted of any oocyte-derived maternal SMCX protein $^{26}$, which may influence X-inactivation patterns in the early embryo. This cross also produced $S m c x^{\Delta / Y}$ males but no $S m c x^{\Delta / \Delta}$ females (Fig. 1a).

To determine if females were perishing due to defects in X-inactivation, we first examined preimplantation mouse embryos. All cells of the female preimplantation mouse embryo undergo imprinted X-inactivation of the paternal X-chromosome ${ }^{27-29}$. We generated $S m c x^{\Delta / \Delta}$ embryonic day (E) 3.5 blastocyst-stage embryos from a cross of Zp3-Cre;Smcx ${ }^{\mathrm{f} / / \Delta}$ females with $S m c x^{\Delta / Y}$ males harboring an X-linked $G f p$ transgene $\left(X-G f p ; S m c x^{\Delta / Y}\right)$. The paternally-transmitted $X$-Gfp transgene permits visual sexing of the embryos since it's only transmitted to female progeny ${ }^{30,31}$. We found that $S m c x^{\Delta / \Delta}$ and control $S m c x^{\mathrm{fl} / \mathrm{fl}}$ female E3.5 embryos displayed similar patterns of imprinted Xist RNA induction and X-linked gene silencing by RNA fluorescent in situ hybridization (FISH) (Extended Data Fig. 1a).

To delineate when $\operatorname{Smcx}^{\Delta / \Delta}$ females were being lost, we next examined E5.5 and E6.5 post-implantation stage embryos. Whereas the control crosses generated the expected distribution of female and male embryos, the test cross yielded significantly fewer E5.5 and E6.5 $S m c x^{\Delta / \Delta}$ female embryos compared to $S m c x^{\Delta / Y}$ male embryos (Fig. 1b). Consistent with the death of the $S m c x^{\Delta / \Delta}$ female embryos, significantly more embryos were resorbed in the test cross compared to the control crosses. Moreover, the surviving $S m c x^{\Delta / \Delta}$ E5.5 embryos were smaller relative to their heterozygous $\operatorname{Smcx} \mathrm{f}^{\mathrm{f} / \Delta}$ female and hemizygous $\operatorname{Smc} x^{\Delta / \mathrm{Y}}$ male littermates (Extended Data Fig. 1b).

We hypothesized that the under-representation of E5.5 Smcx ${ }^{\Delta / \Delta}$ embryos is due to defective random X-inactivation, which begins between E5.0-E5.25 stage of embryogenesis ${ }^{32,33}$. 
We therefore examined Xist RNA expression in epiblasts of surviving E5.5 Smcx ${ }^{\Delta / \Delta}$ female embryos. Compared to $S m c x^{\mathrm{fl} / \mathrm{fl}}$ and $S m c x^{\mathrm{fl} / \Delta}$ genotypes, many nuclei in E5.5 $S m c x^{\Delta / \Delta}$ embryonic epiblasts lacked Xist RNA coating by fluorescence in situ hybridization (FISH) (Fig. 1c-d). Moreover, many $S m c x^{\Delta / \Delta}$ nuclei with Xist RNA coating exhibited qualitatively weaker Xist RNA foci compared to the control $S m c x^{\mathrm{fl} / f \mathrm{l}}$ and $S m c x^{\mathrm{fl} / \Delta}$ nuclei. To test if the deficiency in Xist RNA coating is due to a failure of differentiation of the pluripotent epiblast progenitor cells, we concurrently assayed expression of the REX1 protein by immunofluorescence (IF). REX1 marks pluripotent epiblast progenitor cells in E3.5 embryos and is rapidly downregulated when these cells differentiate ${ }^{33}$, which is accompanied by the onset of random $\mathrm{X}$-inactivation ${ }^{33,34}$. Unlike E3.5 embryos, the E5.5 control and $S m c x^{\Delta / \Delta}$ epiblasts were devoid of REX1 expression, indicating that they had undergone differentiation (Fig. 1c). In agreement with the RNA FISH data, RT-qPCR also revealed significantly reduced Xist RNA expression but similar Rexl RNA levels in E5.5 $S m c x^{\Delta / \Delta}$ compared to $S m c x^{\mathrm{fl} / \mathrm{fl}}$ and $S m c x^{\mathrm{fl} / \Delta}$ epiblasts (Fig. 1e).

To evaluate $\mathrm{X}$-inactivation in vitro, we next derived $S m c x^{\mathrm{fl} / f 1}, S m c x^{\mathrm{fl} / \Delta}$, and $S m c x^{\Delta / \Delta}$ embryonic stem cells (ESCs). ESCs can be differentiated into epiblast-like cells (EpiLCs) ${ }^{35}$,

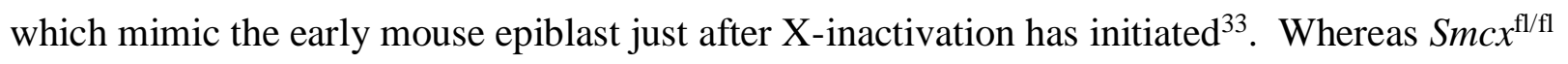
and $S m c x^{\mathrm{fl} / \Delta}$ EpiLCs displayed Xist RNA expression and coating, $S m c x^{\Delta / \Delta}$ EpiLCs were deficient in both (Fig. 1f-h; Extended Data Fig. 1c). Thus, SMCX is required for Xist RNA induction in vivo and in vitro.

\section{Dose-dependent requirement of Smcx in X-linked gene silencing}

We next asked if SMCX is required for X-linked gene silencing, by examining heterozygous $S m c x^{\mathrm{fl} / \Delta}$ epiblasts and EpiLCs, which display robust Xist RNA expression and coating (Fig. 1c-h). Due to potential cell-to-cell and gene-to-gene variability in silencing ${ }^{14}$, we 
employed RNA FISH to compare the expression of eight different genes distributed across the X-chromosome in $S m c x^{\mathrm{fl} / / \mathrm{fl}}$ and $S m c x^{\mathrm{fl} / \Delta}$ E5.5 embryonic epiblasts and EpiLCs (Fig. 2a; Extended Data Fig. 2a). A subset of the tested genes, Lamp2, Mecp2, and Atrx, were expressed from the Xist RNA-coated X-chromosome in significantly more $S m c x^{\mathrm{fl} / \Delta}$ E5.5 embryonic epiblast and EpiLC nuclei compared to $S m c x^{\mathrm{fl} / \mathrm{fl}}$ nuclei (Fig. 2a). These three genes were also transcribed from the Xist RNA-coated X-chromosome in significantly more $X^{\Delta \mathrm{Tsix}} Y$ differentiating EpiLCs compared to $X^{\Delta \text { Tsix }} X$ females (Extended Data Fig. 2b-c). Together, the results in Figs. 1-2 demonstrate that the female-specific dose of SMCX is not only required for Xist RNA expression but also for efficient silencing of X-linked genes once Xist RNA is induced.

We next hypothesized that the $S m c x^{\mathrm{fl} / \Delta}$ cells that displayed inefficient silencing express a reduced level of SMCX than the $S m c x^{\mathrm{fl} / \Delta}$ cells with more stringent silencing of X-linked genes. Although Smcx escapes X-inactivation, expression of Smcx from the inactive-X is lower than from the active- $\mathrm{X}^{21,22}$ (Extended Data Fig. 2d). Therefore, $S m c x^{\mathrm{fl} / \Delta}$ cells harboring the $S m c x^{\mathrm{fl}}$ allele on the inactive-X are expected to express reduced SMCX than those with the Smcx fl allele on the active-X. If SMCX functions as a dose-dependent regulator of X-linked gene silencing, then the former group of cells would be more susceptible to X-inactivation defects than the latter (schematic in Fig. 2b).

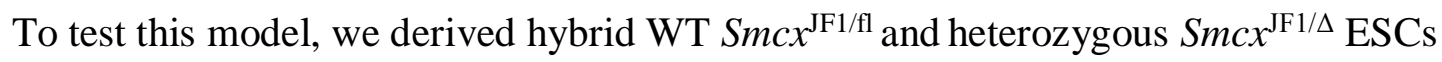
containing X-chromosomes from divergent strains of mice. In these cells, the X-chromosome harboring the $S m c x^{\mathrm{JF} 1}$ allele $\left(\mathrm{X}^{\mathrm{JF} 1}\right)$, which encodes a WT SMCX protein, is derived from the Mus molossinus JF1 strain, and the X-chromosome with the $S m c x^{\mathrm{fl}}$ or the $S m c x^{\Delta}$ allele $\left(X^{\mathrm{fl} I \mathrm{Smcx}}\right.$ and $X^{\Delta \mathrm{Smcx}}$, respectively) is derived from the Mus musculus laboratory strain. We generated EpiLCs from these hybrid ESCs, and exploited single nucleotide polymorphisms (SNPs) in Xist on the 
two $\mathrm{X}$-chromosomes to mark the $\mathrm{X}$ that was chosen for inactivation by allele-specific Xist RNA FISH (see Methods; Extended Data Fig. 3a). Xist RNA is upregulated from and coats the Xchromosome that is selected for inactivation ${ }^{9,10,36}$. We concurrently evaluated the expression of the three genes that were not efficiently silenced in Smcx ${ }^{\mathrm{f} / / \Delta}$ EpiLCs, Lamp2, Mecp2, and Atrx, via RNA FISH (see Fig. 2a). In the control Smcx ${ }^{\mathrm{JF1} / \mathrm{fl}}$ EpiLCs, the three genes were silenced with similar efficiency irrespective of which X-chromosome was Xist RNA coated (Fig. 2c), as expected, since both Xs harbor active $S m c x$ alleles that generate WT protein. By contrast, in $S m c x^{\mathrm{fl} / \Delta}$ EpiLCs all three genes were significantly more likely to be expressed from the Xist RNA-coated WT $X^{\mathrm{JF} 1}$ as compared to the $X^{\Delta \mathrm{Smcx}}$ (Fig. 2c; Extended Data Figs. 3b-f and 4). These data reiterate a dose-dependent function of SMCX in X-linked gene silencing during Xinactivation.

\section{Sufficiency of SMCX in Xist RNA induction and X-linked gene silencing}

Having established a dose-dependent requirement of SMCX in X-linked gene silencing, we next tested the sufficiency of Smcx in inducing X-inactivation in male cells. Strikingly, ectopic expression of a mouse $\operatorname{Smcx}(T g-m S m c x)$ as well as a human SMCX $(T g-h S M C X)$ transgene resulted in induction of Xist RNA in $>40 \%$ of $X Y$ male ESCs (Fig. 3a). We additionally tested if the Xist RNA induction required the demethylase activity of SMCX. Expression of a point-mutant that abolishes the demethylase function of SMCX ( $\mathrm{Tg}$ $h \operatorname{SMCX}(H 514 A))^{15}$ was not able to induce Xist RNA in the $X Y$ male ESCs (Fig. 3a). Similarly, ectopic expression of the closely related SMCX homolog, SMCY/KDM5D (Tg-hSmcy), which maps to the Y-chromosome and is itself capable of demethylating $\mathrm{H} 3 \mathrm{~K} 4 \mathrm{me} 2 / 3^{15}$, was also insufficient to activate Xist RNA expression (Fig. 3a). Thus, although both SMCX and SMCY can demethylate $\mathrm{H} 3 \mathrm{~K} 4 \mathrm{me} 2 / 3$, only SMCX is able to induce Xist in an enzymatic activity- 
dependent manner (see Extended Data Fig. 5 and Supplemental Fig. 1). Moreover, more than $75 \%$ of the Xist RNA-coated Tg-mSmcx and Tg-hSMCX XY ESCs also silenced the three Xlinked genes that are not efficiently silenced in $S m c x^{\mathrm{fl} / \Delta}$ female EpiLCs, Lamp2, Mecp2, and Atrx (Figs. 3b and 2a), supporting the dual functions of SMCX in Xist induction and X-linked gene silencing.

We next took advantage of the sensitized background of $X^{\Delta \text { Tsix }} Y$ ESCs to further investigate the dose-dependency of SMCX in inducing Xist expression and X-linked gene silencing. In differentiating $X^{\Delta T s i x} Y$ male EpiLCs, some but not all cells ectopically induce Xist RNA due to a deletion in the Xist antisense repressor $T$ six ${ }^{14}$; moreover, only a subset of the cells with Xist RNA coats silence X-linked genes (outlined in Fig. 3c). We first tested if SMCX overexpression could increase the frequency of Xist RNA-coated $X^{\Delta \mathrm{Tsix}} Y$ nuclei. Indeed, compared to the parent $X^{\Delta \mathrm{Tsix}} Y$ cells, d2 differentiated EpiLCs ectopically expressing mouse and human SMCX (Tg-mSmcx; $X^{\Delta \mathrm{Tsix}} Y$ and $T g-h S m c x ; X^{\Delta \mathrm{Tsix}} Y$, respectively) displayed a significant increase in the percentage of Xist RNA-coated nuclei (Fig. 3d-e and Extended Data Fig. 6a). Cells ectopically expressing the enzymatically-inactive SMCX protein $(\mathrm{Tg}$ $\left.h S M C X(H 514 A) ; X^{\Delta T s i x} Y\right), \operatorname{SMCY}\left(T g-h S M C Y ; X^{\Delta \operatorname{Tsix}} Y\right)$, or lacking the Y-chromosome $\left(X^{\Delta \operatorname{Tsix}} O\right)$, on the other hand, exhibited a similar frequency of Xist RNA-coated X-chromosomes as the $X^{\Delta \mathrm{Tsix}} Y$ cells (Fig. $3 \mathrm{~d}$ ). Reciprocally, the loss of SMCX in $X^{\Delta \mathrm{Tsix} ; \Delta \mathrm{Smcx}} Y$ and $X^{\Delta \mathrm{Tsix} ; \Delta \mathrm{Smcx}} O$ cells reduced the incidence of ectopic Xist RNA coating (Fig. 3d). Xist RNA levels quantified by RTqPCR were concordant with the RNA FISH data (Fig. 3e).

In addition to Xist RNA induction, we examined the impact of SMCX in the silencing of $\mathrm{X}$-linked genes in $X^{\Delta \mathrm{Tsix}} Y$ cells. The overexpression of SMCX in differentiated $X^{\Delta \mathrm{Tsix}} Y$ EpiLCs resulted in an increased frequency of X-linked gene silencing (Fig. 3f; Extended Data Fig. 6b-c 
and Extended Data Table 1). Increased expression of the mutant SMCX (H514A) or SMCY again resulted in a gene silencing pattern similar to the parental $X^{\Delta \mathrm{Tsix}} Y$ cells. Conversely, the absence of SMCX in differentiating $X^{\Delta \mathrm{Tsix} ; \Delta \operatorname{Smcx}} Y$ and $X^{\Delta \mathrm{Tsix} ; \Delta \operatorname{Smcx}} O$ EpiLCs significantly reduced the percentage of nuclei with silenced X-linked genes. Moreover, $X^{\Delta \mathrm{Tsix}} O$ and $X^{\Delta \mathrm{Tsix} ; \Delta \mathrm{Smcx}} O$ differentiating EpiLCs recapitulated the pattern of silencing in differentiating $X^{\Delta \mathrm{Tsix}} Y$ and $X^{\Delta \text { Tsix; } \Delta \text { Smcx }} Y$ cells, thus excluding a contribution of $Y$-linked genes in the observed differences.

\section{SMCX dose-dependent histone H3K4 demethylation on the inactive-X}

To test if SMCX directly regulates X-inactivation, we first examined if SMCX is enriched on the inactive-X cytologically. To ensure efficient detection of SMCX, we generated $X X$ female ESCs stably expressing an epitope-tagged SMCX protein (Strep-SMCX). StrepSMCX accumulated on the X-chromosome as Xist RNA began to coat the X-chromosome prior to the differentiation of ESCs into EpiLCs (Fig. 4a). SMCX enrichment was transient and was largely lost by the time the ESCs differentiated into EpiLCs. In addition, only the Xist RNAcoated inactive X-chromosome, but not the active-X, displayed the SMCX accumulation in the female cells (Fig. 4a), indicating that the transient SMCX recruitment is an Xist RNA-dependent process. A similarly transient accumulation of SMCX characterized the onset of Xist RNA accumulation in differentiating $X^{\Delta \mathrm{Tsix}} Y$ EpiLCs. Slower kinetics of the SMCX enrichment on the X-chromosome upon Xist RNA coating in male compared to female cells (Fig. 4a) mirrors the slower induction of Xist RNA in the $X^{\Delta \mathrm{Tsix}} Y$ cells ${ }^{37}$.

SMCX is a member of the KDM5-family of enzymes that demethylate H3K4me2/3 15,38 , which are well-characterized hallmarks of transcriptionally-engaged chromatin ${ }^{17}$. H3K4me2 is excluded from the inactive- $\mathrm{X}^{20}$. To test if SMCX is responsible for H3K4me2/3 exclusion, we examined $\mathrm{H} 3 \mathrm{~K} 4 \mathrm{me} 2$ depletion from the inactive-X as a function of SMCX dose. The percentage 
of nuclei exhibiting depletion of H3K4me2 and H3K4me3 from the Xist RNA-coated Xchromosome increased with increasing SMCX dose (Fig. 4b and Extended Data Fig. 7a). To further probe H3K4me2 occupancy on the inactive-X when SMCX dose is modulated, we performed H3K4me2 ChIP-Seq in differentiating $X^{\Delta \mathrm{Tsix}} Y, X^{\Delta \mathrm{Tsix} ; \Delta \mathrm{Smcx}} Y$, and $T g-S m c x ; X^{\Delta \mathrm{Tsix}}$ EpiLCs. We found an inverse correlation between cellular SMCX levels and H3K4me2 genome-wide (Fig. 4c-d). The increase in H3K4me2 upon the loss of SMCX was more pronounced on the X-chromosome compared to the autosomes, suggesting that H3K4me2 occupancy on the X-chromosome is more sensitive to a reduced SMCX dose compared to autosomes (Fig. 4c and Extended Data Fig. 7b-d). To investigate the relationship of H3K4me2 and transcription, we examined $\mathrm{H} 3 \mathrm{~K} 4 \mathrm{me} 2$ at TSSs of the eight X-linked genes profiled by RNA FISH in Fig. 2a. The three genes that were not silenced stringently in female $\operatorname{Smcx} \mathrm{x}^{\mathrm{f} / \Delta}$ cells, Lamp2, Mecp2, and Atrx, showed the greatest change in promoter H3K4me2 levels as a function of SMCX dose (Fig. 4e and Extended Data Fig. 8a). Thus, X-linked gene silencing by SMCX entails the removal of $\mathrm{H} 3 \mathrm{~K} 4 \mathrm{me} 2$ from promoters.

The impact of SMCX dose on H3K4me2 levels persisted even after the transient SMCX enrichment on the X-chromosome dissipated (Fig. 4a-e). Although the cytological accumulation of SMCX on the inactive-X disappeared in differentiating EpiLCs (Fig. 4a), by ChIP-Seq residual SMCX protein was nevertheless detected at gene regulatory elements such as promoters and distal DNaseI hypersensitive sites (DHS) at X-linked genes (Extended Data Fig. 8a-b). At the SMCX-enriched promoters, loss of SMCX led to a significant increase in H3K4me2 at both the X-chromosome as well as on a genome-wide scale (Extended Data Fig. 8c). These results suggest that SMCX is transiently recruited onto the X-chromosome at the onset of X-inactivation 
and that the SMCX-dependent removal of $\mathrm{H} 3 \mathrm{~K} 4 \mathrm{me} 2$ at promoters is maintained after SMCX dislodges from X-linked genes.

We then sought to probe the mechanism by which SMCX induces Xist expression. In contrast to the TSS-surrounding SMCX ChIP-Seq signals found at most X-linked genes, at the Xist locus, SMCX is enriched $\sim 1.5 \mathrm{~kb}$ downstream of the TSS (Fig. 5a-b and Extended Data Fig. 8a). We previously reported an enhancer-like regulatory element that promotes Xist expression in this region ${ }^{39}$. Importantly, SMCX reverses $\mathrm{H} 3 \mathrm{~K} 4 \mathrm{me} 2 / 3$ and leaves intact $\mathrm{H} 3 \mathrm{~K} 4 \mathrm{me}{ }^{15}$, which is a hallmark of transcriptional enhancers ${ }^{40,41}$. Active enhancers are also enriched in $\mathrm{H} 3 \mathrm{~K} 27$ acetylation $(\mathrm{H} 3 \mathrm{~K} 27 \mathrm{ac})^{42}$. We therefore tested $\mathrm{H} 3 \mathrm{~K} 4 \mathrm{me} 1 / 2 / 3$ and $\mathrm{H} 3 \mathrm{~K} 27 \mathrm{ac}$ occupancy at the putative Xist enhancer by ChIP-qPCR.

Consistent with the previous study ${ }^{39}$, we noted that the SMCX-enriched region downstream of the Xist TSS indeed displayed enhancer-like chromatin signature of high H3K4me1 and lower H3K4me2/3 in d2 differentiated $X^{\Delta \mathrm{Tsix}} Y$ EpiLCs (Fig. 5c). Conversely, the TSS-proximal region of Xist was decorated with higher H3K4me2/3 and lower H3K4me1. In the Xist enhancer region, SMCX overexpression led to a further increase in H3K4me1 and a decrease in $\mathrm{H} 3 \mathrm{~K} 4 \mathrm{me} 2 / 3$, and was accompanied by appearance of the active enhancer mark H3K27ac. In contrast, at the Xist promoter, which is devoid of SMCX, we observed an increase in the active chromatin signatures H3K4me2/3 and H3K27ac upon SMCX overexpression. When we analyzed the Atrx promoter, which is directly bound by SMCX in the d2 differentiating EpiLCs, SMCX overexpression resulted in the removal of H3K4me2/3. These changes in histone modification levels at the Xist enhancer or the Atrx promoter were not observed when we overexpressed the mutant SMCX-H514A mutant (Fig. 5c), consistent with the demethylase activity-dependent induction of Xist RNAs (Fig. 4). The absence of SMCX resulted in an 
opposite pattern in histone modifications compared to SMCX overexpression, reinforcing the SMCX-dose dependency in both promoter and enhancer regulation (Fig. 5c). Note that Tsix is not expressed in the $X^{\Delta \mathrm{Tsix}} Y$ EpiLCs ${ }^{33}$; therefore, we rule out indirect induction of SMCX via $T$ six suppression. These results support a model in which SMCX directly induces Xist expression via enhancer activation and silences $\mathrm{X}$-linked genes by promoter suppression.

\section{Discussion}

In this study, we show that the histone H3K4me2/3 demethylase SMCX functions in a dose- and enzymatic activity-dependent manner both to induce Xist and, separately, to silence Xlinked genes (Fig. 5d). The higher, biallelic expression of SMCX links the discrete steps that are thought to underlie random $\mathrm{X}$-inactivation ${ }^{43,44}$ : sensing the $\mathrm{X}$-chromosome complement in the cell ('counting' step), inducing expression of Xist ('initiation' step), and silencing of X-linked genes ('establishment' step). All X-chromosome sequence elements necessary and sufficient to induce $\mathrm{X}$-inactivation are thought to reside within the $X$-inactivation center $(X i c)$, which harbors the Xist locus ${ }^{43,44}$. Smcx, however, maps far outside of the Xic, near the telomeric end of the Xchromosome. The results herein argue against the primacy of the Xic and instead position Smcx at or near the top of the molecular hierarchy leading to random X-inactivation (Fig. 5d).

$\mathrm{X}$-inactivation is postulated to have evolved as a consequence of the deterioration of the Y-chromosome ${ }^{2}$, which was once homologous to the X-chromosome in the common ancestor of therian mammals ${ }^{45}$. By extension, the presence of active $\mathrm{Y}$-linked genes is believed to have driven escape from inactivation of the $\mathrm{X}$-linked homologs to adjust the dose of $\mathrm{X}$-Y gene pairs between the sexes ${ }^{46}$. Our data instead demonstrate that X-Y homologs can be functionally distinct, with the X-linked copy having evolved a female-specific function to cause $\mathrm{X}$ - 
bioRxiv preprint doi: https://doi.org/10.1101/175174; this version posted August 11,2017 . The copyright holder for this preprint (which was not certified by peer review) is the author/funder, who has granted bioRxiv a license to display the preprint in perpetuity. It is made available under aCC-BY-NC-ND 4.0 International license.

inactivation. Escape from X-inactivation may therefore be evolutionarily driven in part by the requirement of the $\mathrm{X}$-linked homologs in X-chromosome dosage compensation. 


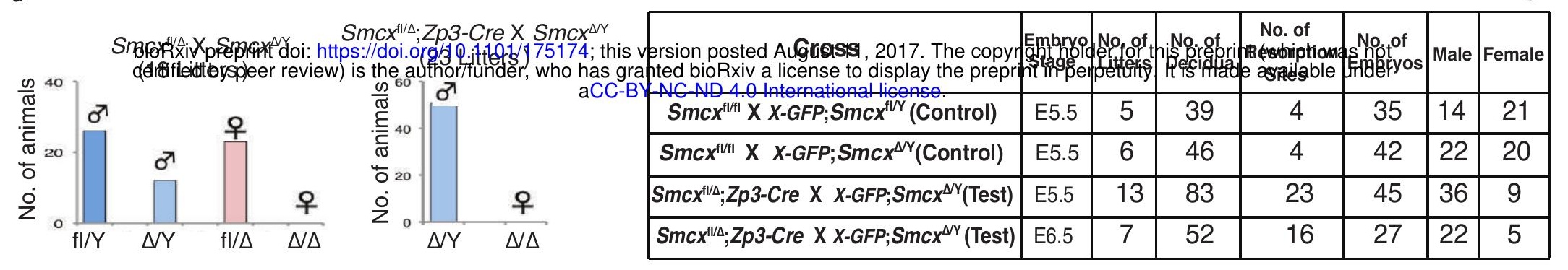
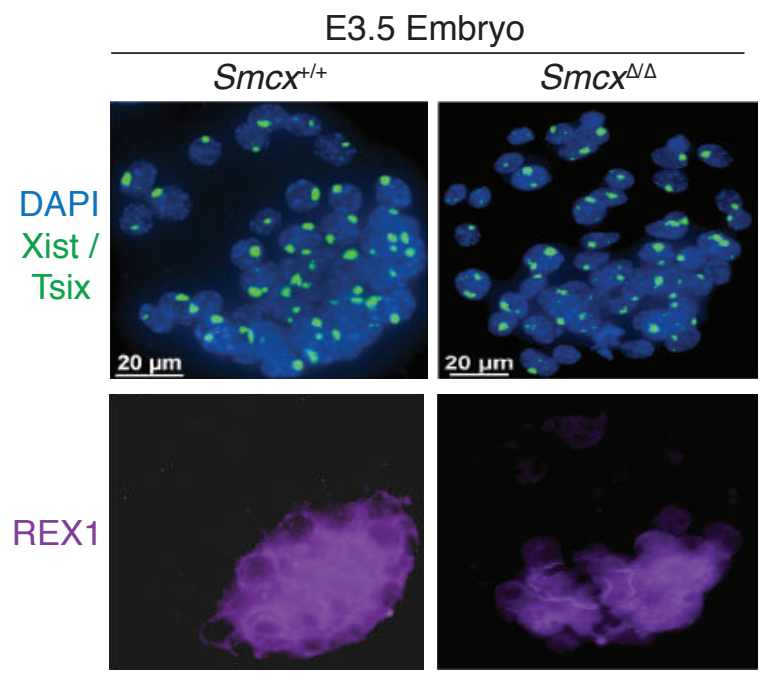

E5.5 Epiblast
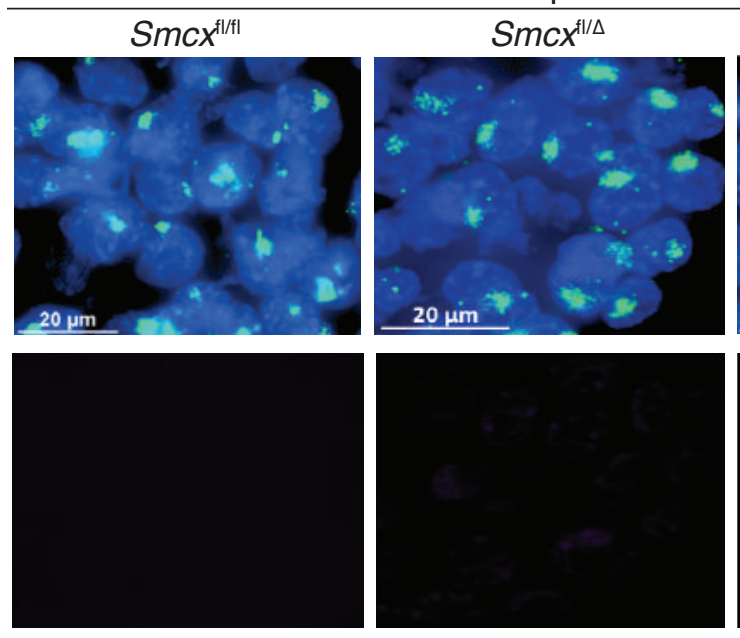
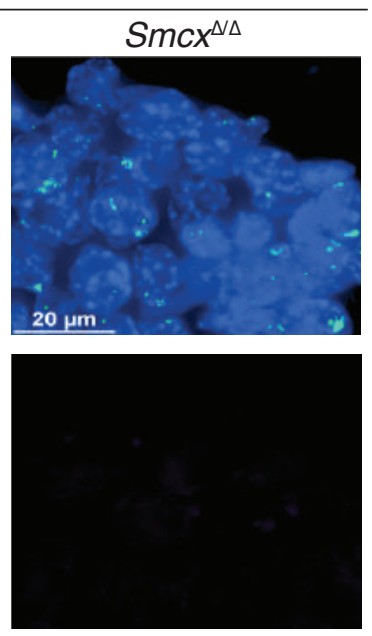

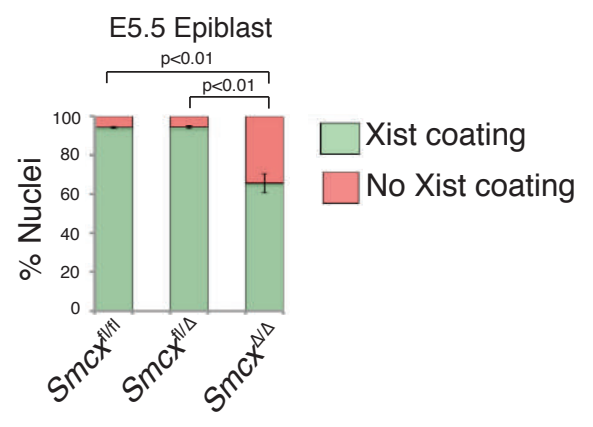

$S m c x^{|t||f|}$

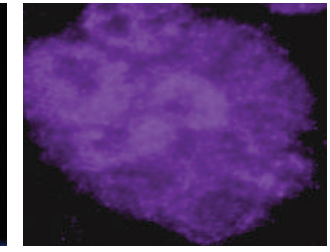

$20 \mu \mathrm{m}$

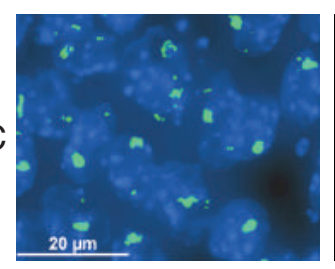

DAPI Xist /Tsix

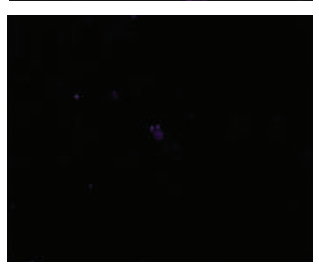

REX1

乌

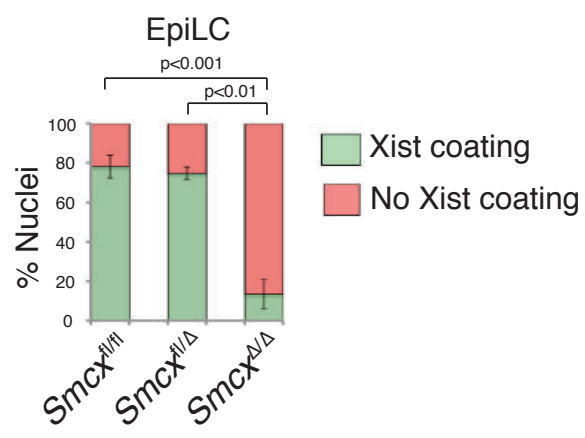

e
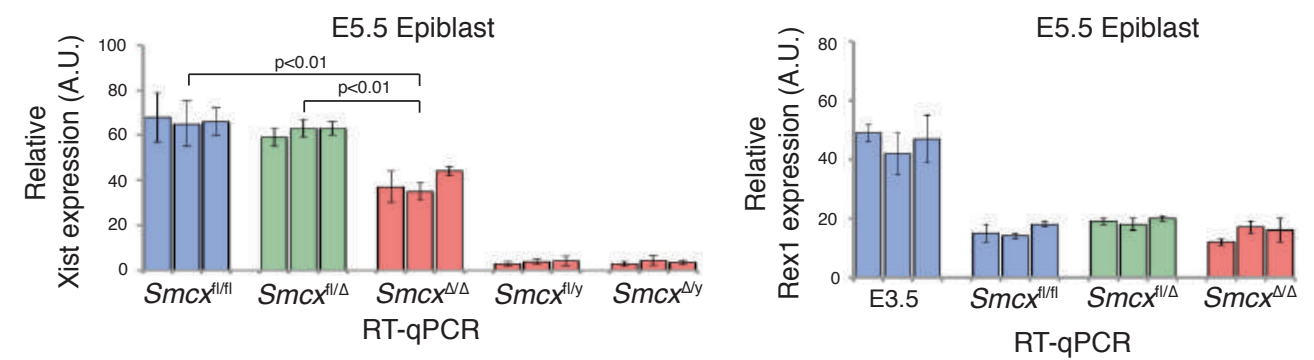

RT-qPCR

\section{$S m c X^{\Delta \Delta}$}

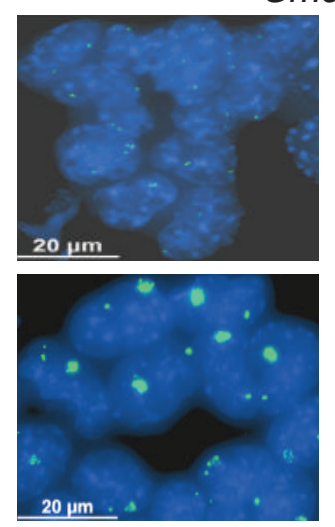

DAPI Xist /Tsix
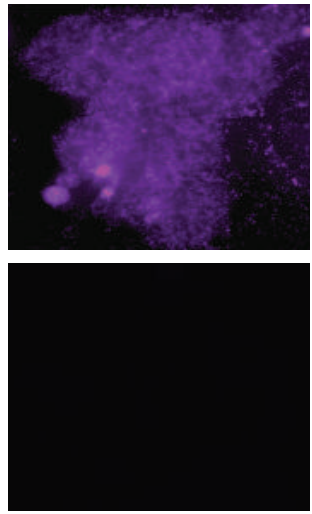

REX1
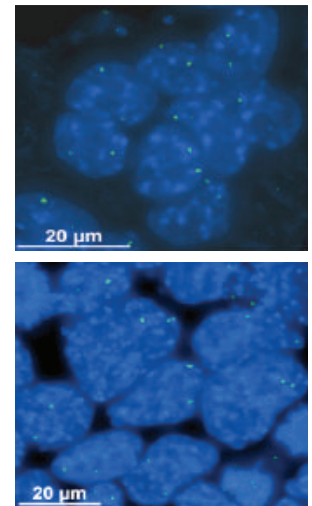

DAPI Xist / Tsix
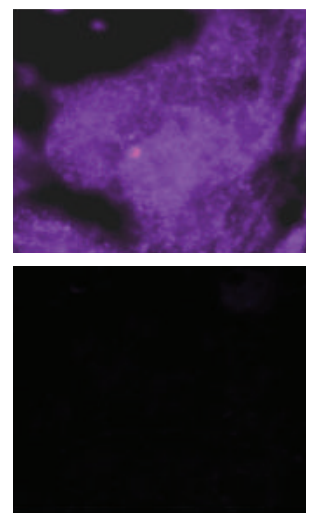

REX1

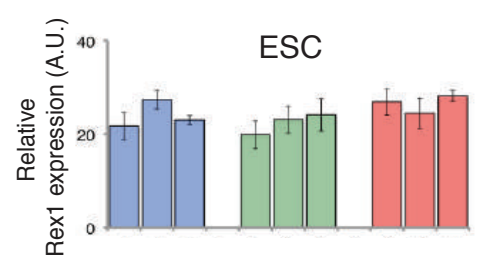

EpiLC
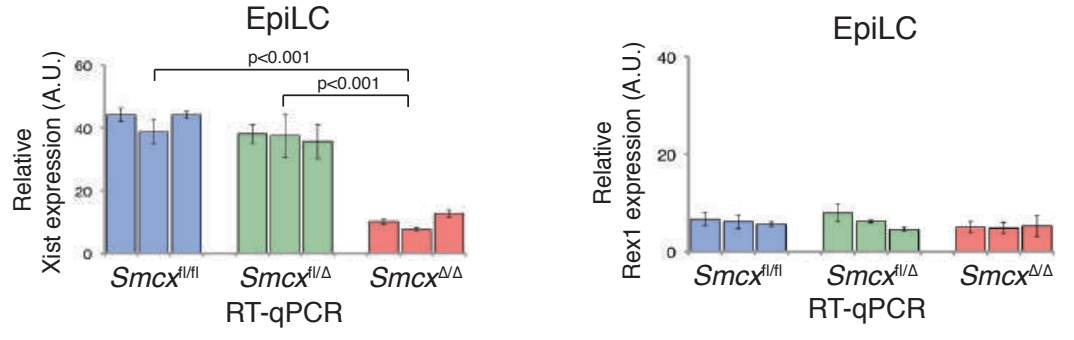
Figure 1: Defective X-inactivation in $\operatorname{Smcx}^{\Delta / \Delta}$ female embryos and EpiLCs. a-b, Live-born animals and E5.5 and E6.5 embryos obtained from intercrosses of Smcx-mutant animals. c, RNA FISH detection of Xist/Tsix RNAs coupled with IF staining for the pluripotency marker REX1 in representative E5.5 epiblasts. Rex1 downregulation marks differentiating pluripotent epiblast progenitors $^{34,47}$. Nuclei are stained blue with DAPI. d, Quantification of Xist RNA coated nuclei in the E5.5 epiblasts. $\mathrm{n}=150-315$ nuclei in each of 3 embryos/genotype. e, Relative quantification of Xist and Rex1 RNAs by RT-qPCR in E5.5 epiblasts. n=3 embryos/genotype, each analyzed in triplicate. f, Xist/Tsix RNAs and REX1 profiling in representative ESCs and EpiLCs. g, Quantification of Xist RNA-coated EpiLCs. n=100 nuclei from EpiLCs differentiated from each of 3 independent ESC lines/genotype in $\mathbf{f - g}$. $\mathbf{h}$, Relative quantification of Xist and Rex1 RNAs by RT-qPCR in the ESCs and EpiLCs. n=3 independent ESC lines differentiated into EpiLCs/genotype, each analyzed in triplicate. Error bars, standard error. $p$ values, Welch's two-sided t-test. 

aCC-BY-NC-ND 4.0 International license.5.5 Epiblast

Xist RNA coat

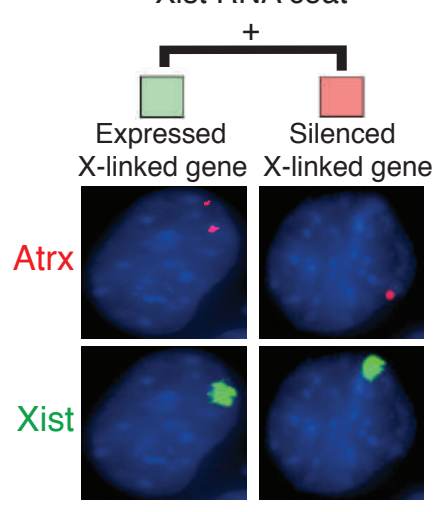

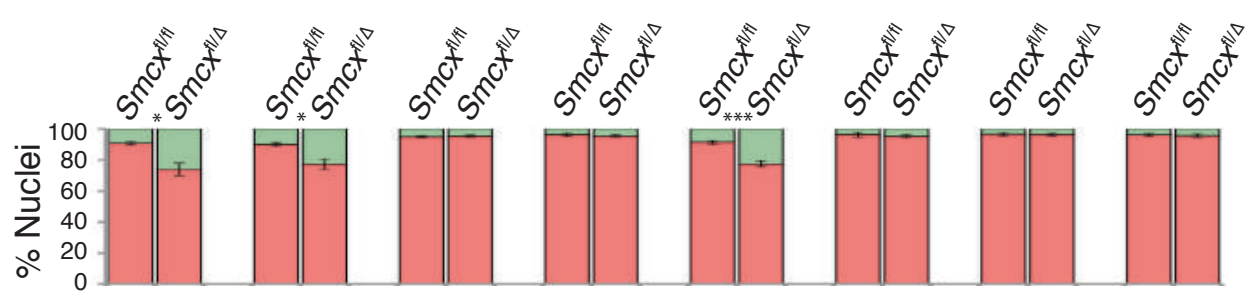

EpiLC

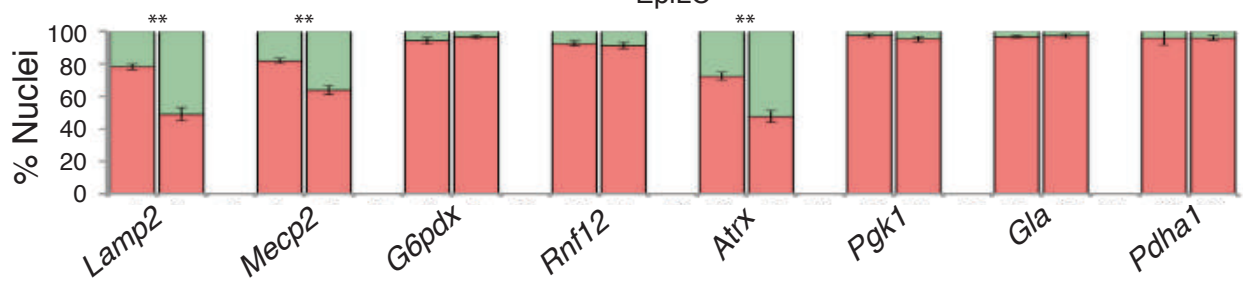

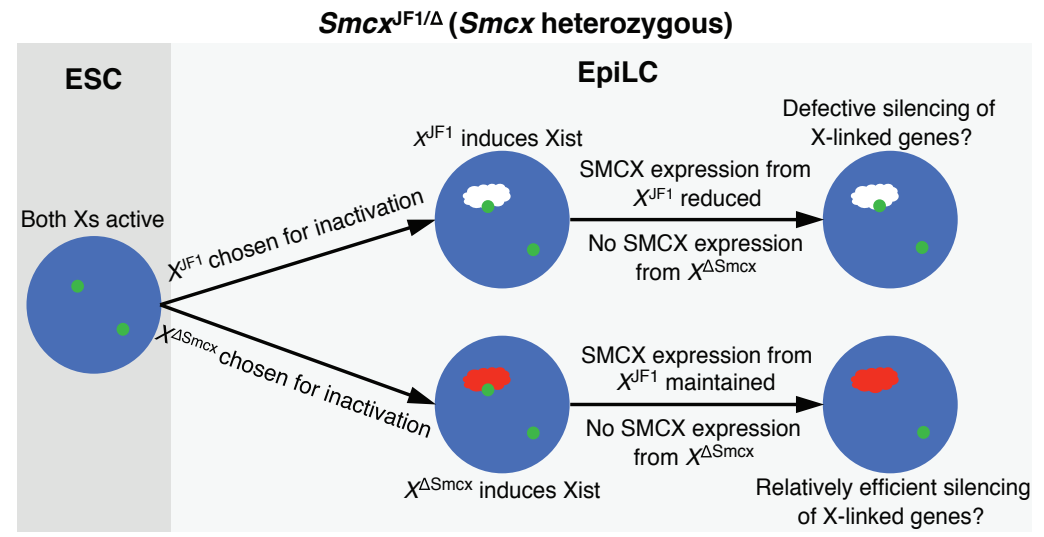

Allelic Xist RNA coat $+\begin{gathered}\text { Expressed } \\ \text { X-linked gene }\end{gathered}$

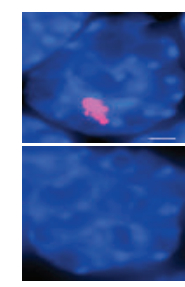

Xist: $X^{\text {Xismcx }}$ or $X^{\Delta \mathrm{Smcx}}$

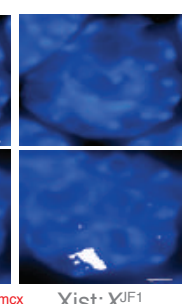

Xist: $X^{\mathrm{JF} 1}$

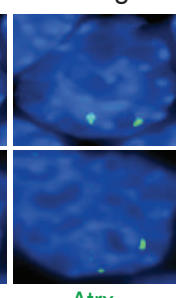

Atrx
Allelic Xist RNA coat +
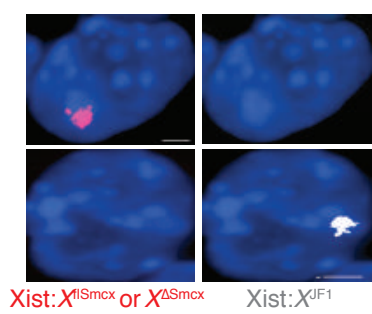

Silenced

X-linked gene

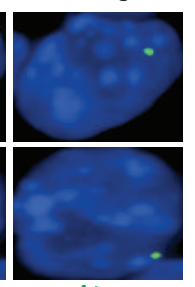

Atrx

Xist RNA coat

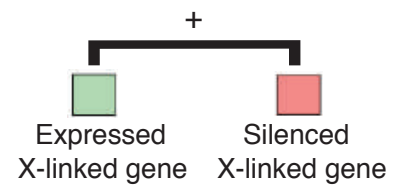

EpiLCs

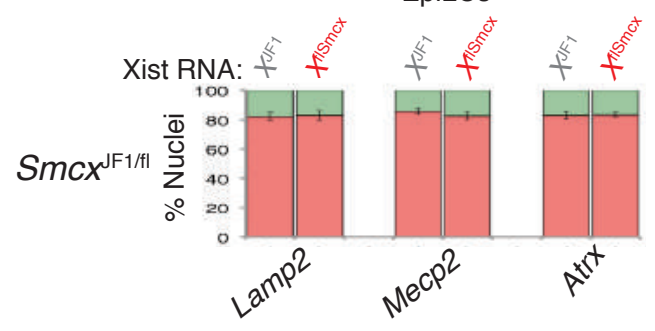

EpiLCs

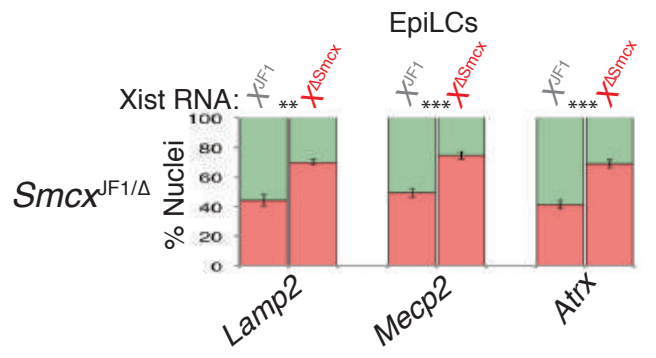


Figure 2: SMCX dose-dependent X-linked gene silencing in $\operatorname{Smcx}{ }^{\mathrm{f} / / \Delta}$ EpiLCs. a, RNA FISH quantification of X-linked gene expression. Left, a representative nucleus with nascent transcripts of one of the genes surveyed, Atrx, in red and Xist RNA in green. $\mathrm{n}=86-141$ nuclei in each of 3 embryonic epiblasts/genotype; and, n=100 nuclei from EpiLCs differentiated from each of 3 independent ESC lines/genotype. Error bars, standard error. *, $p \leq 0.05 ; * *, p<0.01$; ***, $p<0.001$, Welch's two-sided t-tests. b, Schematic of possible patterns of X-linked gene expression by RNA FISH in $S m c x^{\mathrm{JF} 1 / \Delta}$ EpiLCs. c, Top, representative example of the RNA FISH outcome in $S m c x^{\mathrm{JF} 1 / \Delta}$ EpiLCs. Bottom, quantification of expression of three X-linked genes coincident with allele-specific Xist RNA coating in $S m c x^{\mathrm{JF} 1 / f 1}$ or $S m c x^{\mathrm{JF} 1 / \Delta}$ EpiLCs. $\mathrm{n}=100$ nuclei from EpiLCs differentiated from each of 3 independent ESC lines/genotype. 


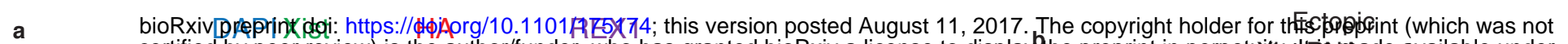

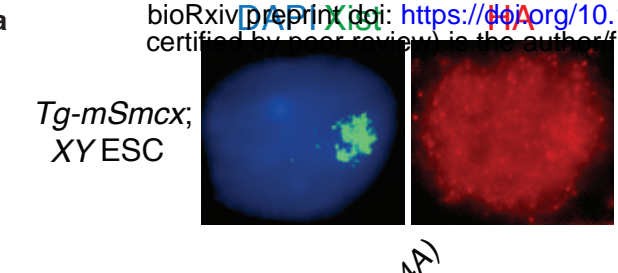

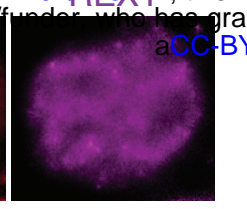
ranted bioRxiv a license to display'the preprint in perpetuxilst' BY-NC-ND 4.0 International license.

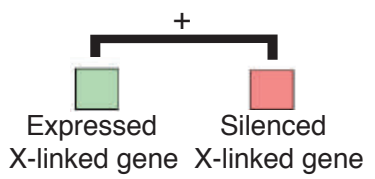

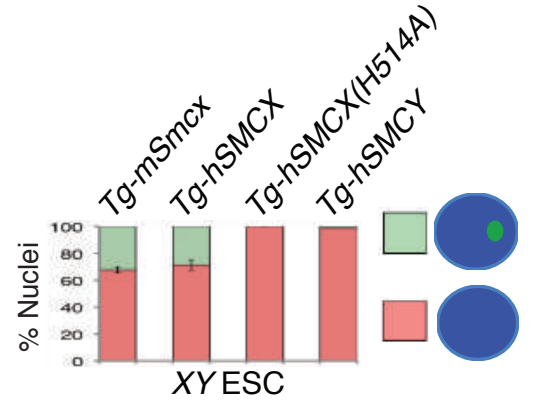

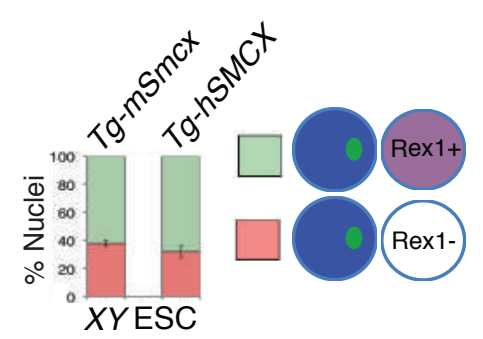

Tg-mSmcx; $X Y$ ESC

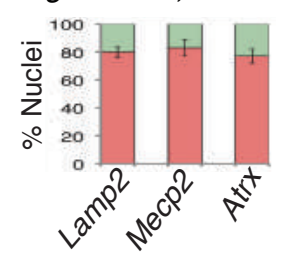

Tg-hSMCX; XY ESC

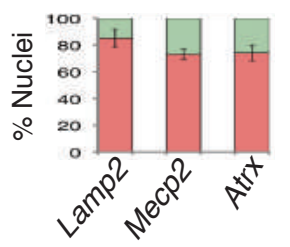

。

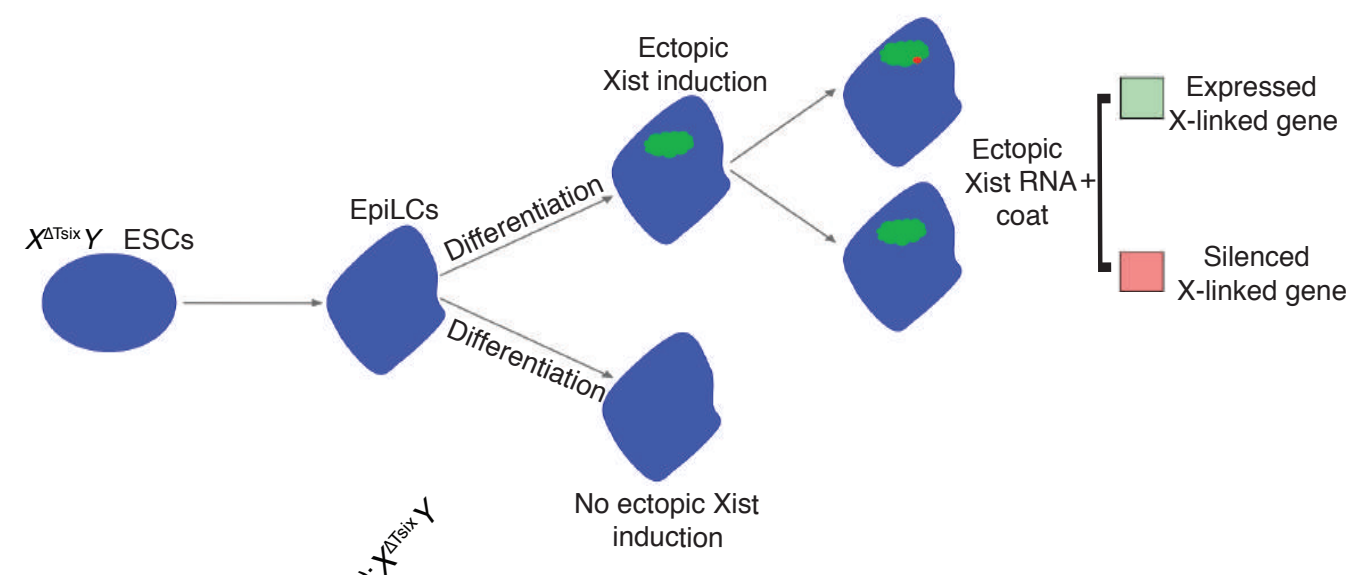

d

Xist RNA coat

No Xist RNA coat
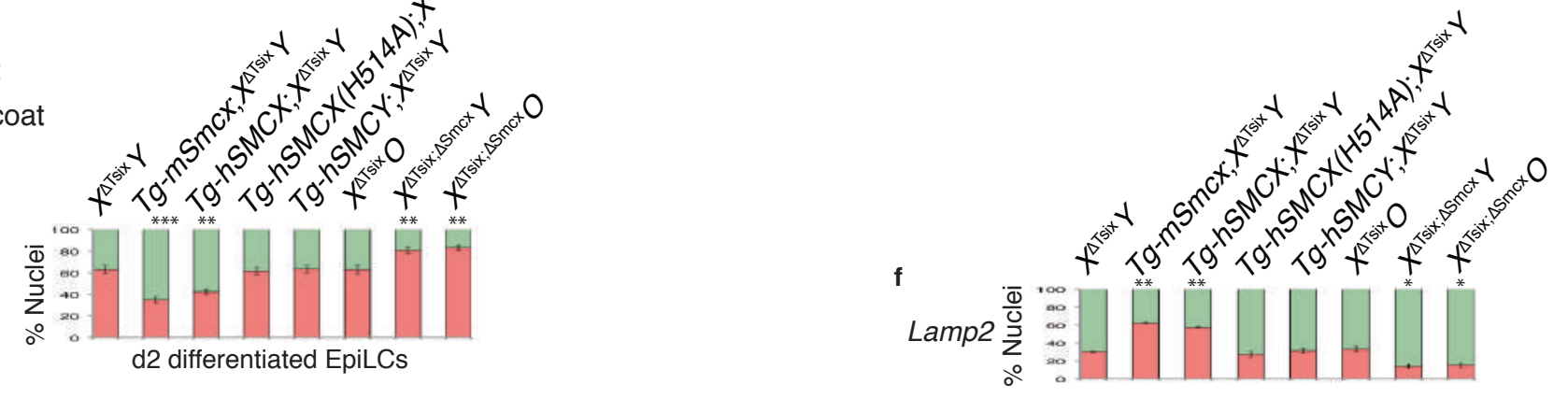

e

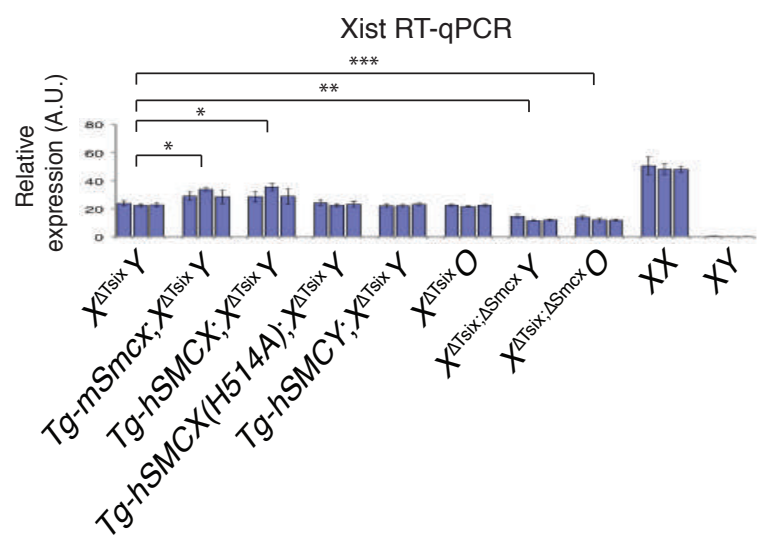

Rex1 RT-qPCR
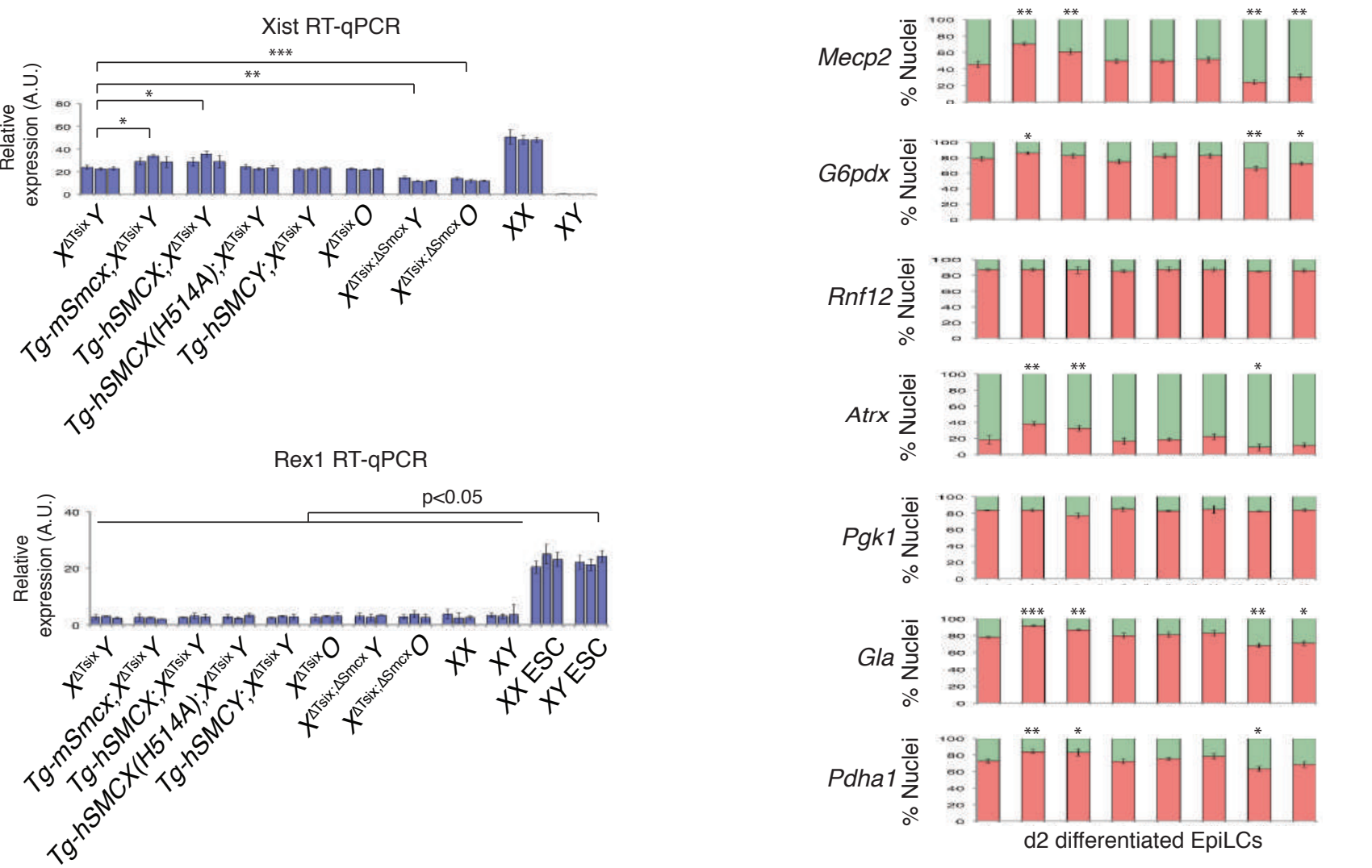

d2 differentiated EpiLCs

Fig.3 


\section{Figure 3: Ectopic Xist RNA induction upon SMCX overexpression in male cells. a,}

Representative example (top) and quantification (bottom) of RNA FISH detection of Xist/Tsix RNAs (green) coupled with IF staining for the HA epitope tag (red) and REX1 (purple) in XY male ESCs expressing an inducible mouse HA-SMCX transgene (Tg-mSmcx); human HASMCX transgene (Tg-hSMCX); an HA-SMCX mutant transgene lacking demethylase activity $(T g-h S M C X(H 514 A))$; and, an HA-SMCY transgene $(T g-h S M C Y) . n=100$ nuclei from each of 3 independent ESC lines. b, Expression of the X-linked genes Lamp2, Mecp2, and Atrx from the Xist RNA-coated X-chromosome in the transgenic $X Y$ ESCs. $\mathrm{n}=100$ nuclei from each of 3 independent ESC lines. c, Schematic of ectopic Xist RNA coating and X-linked gene silencing during differentiation of $X^{\Delta \mathrm{Tsix}} Y$ ESCs. d, Quantification of ectopic Xist RNA-coated nuclei in d2 differentiated EpiLCs. $n=100$ nuclei from EpiLCs differentiated from each of 3 independent ESC lines/genotype. e, Relative quantification of Xist (top) and Rex1 (bottom) RNAs by RTqPCR in the d2 differentiated EpiLCs. $n=3$ independent ESC lines differentiated into EpiLCs/genotype, each analyzed in triplicate. f, Quantification of X-linked gene expression upon ectopic Xist induction in $\mathrm{d} 2$ differentiated EpiLCs. $\mathrm{n}=100$ nuclei from EpiLCs differentiated from each of 3 independent ESC lines/genotype. Error bars, standard error. *,$p \leq$ $0.05 ; * *, p<0.01 ; * * *, p<0.001$, Welch's t-tests. All samples statistically compared to the percentage in $X^{\Delta \mathrm{Tsix}} Y$ cells (see Extended Data Table 1 for exact values). 

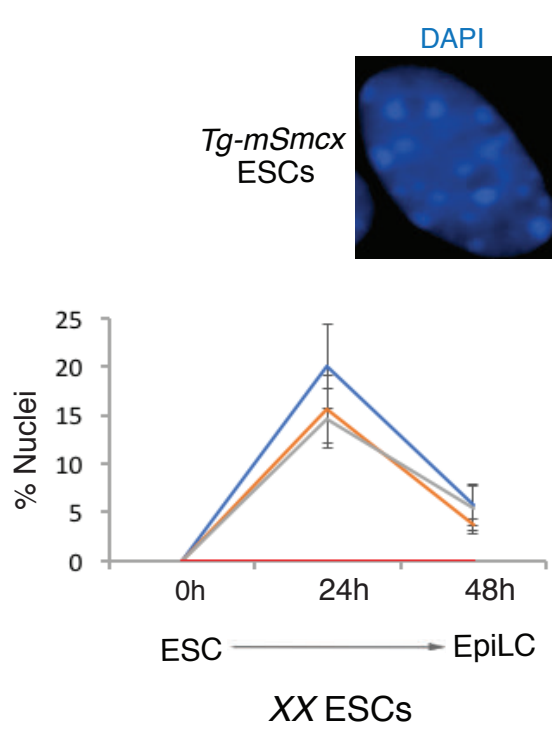

DAPI / Xist RNA

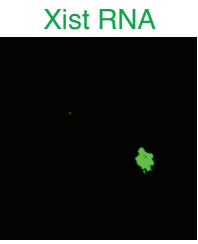

Strep
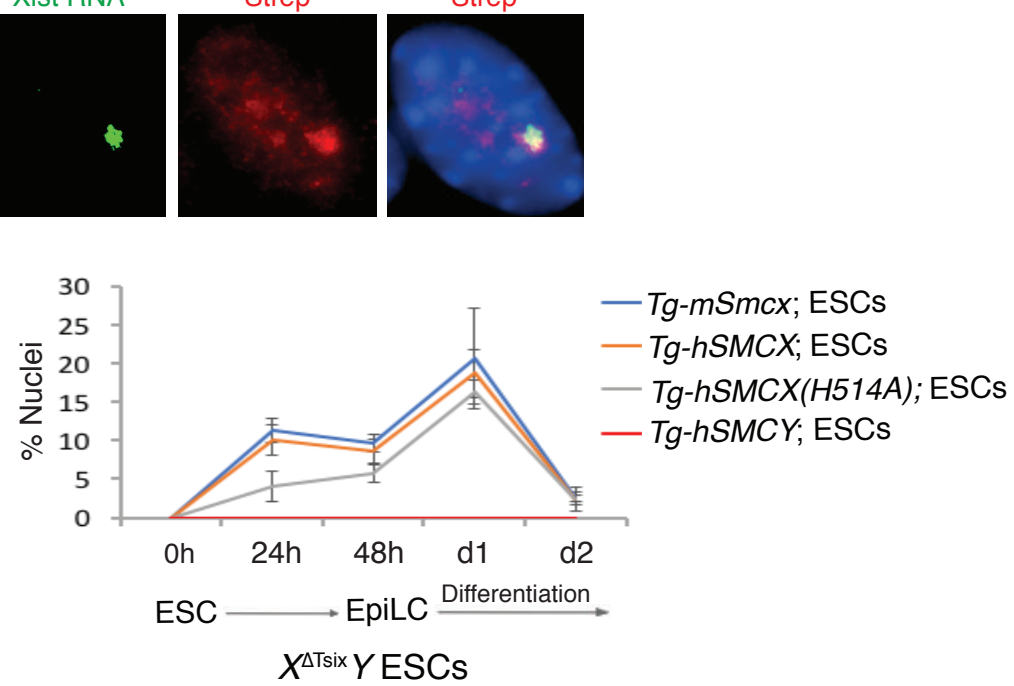

b

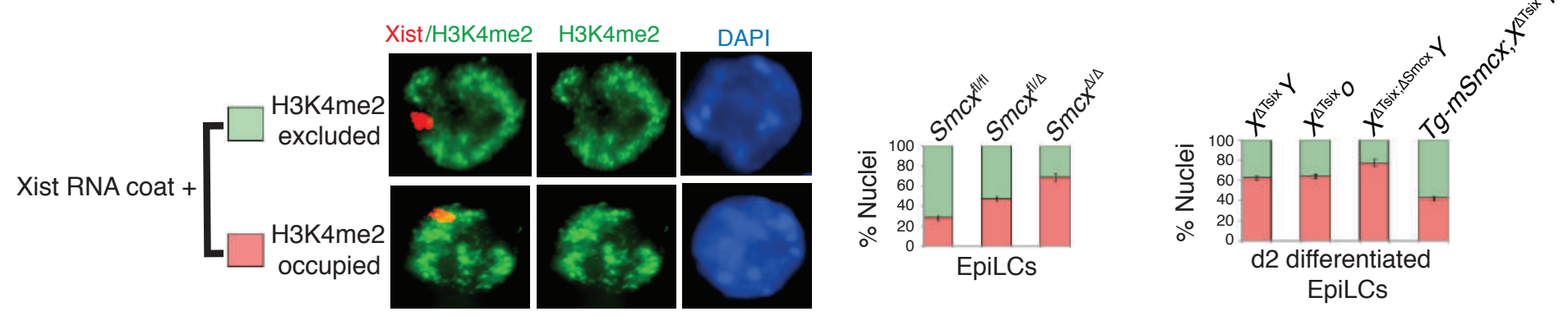

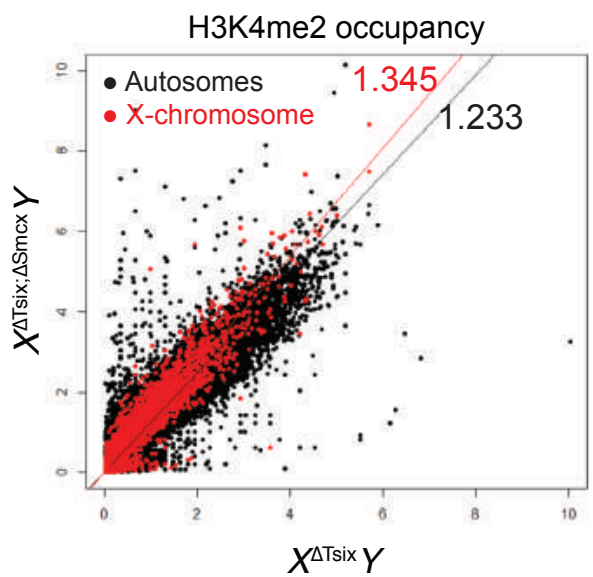

d

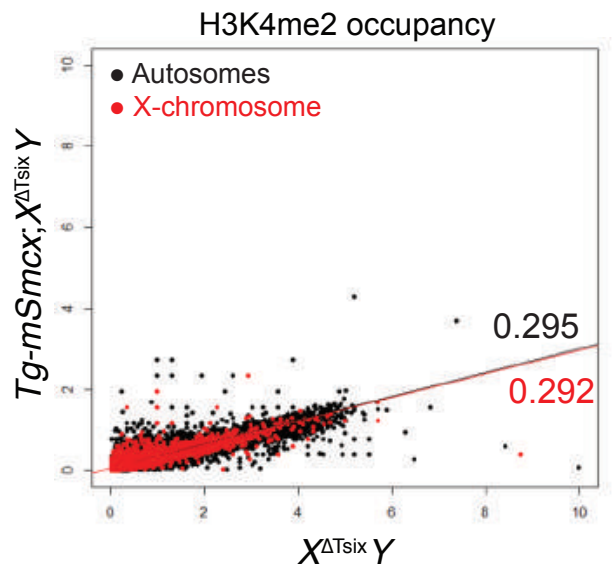

e

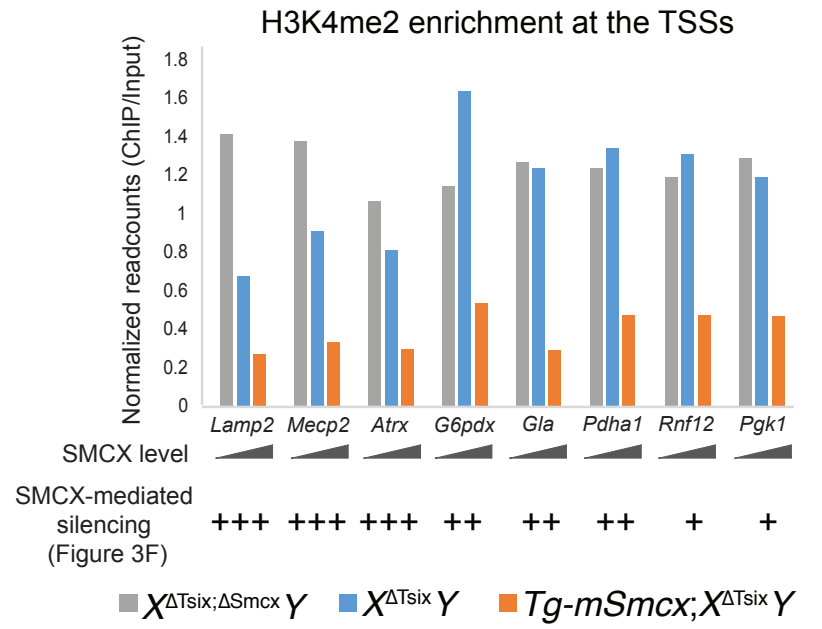


Figure 4: SMCX-dose dependent H3K4me2 exclusion from the inactive X-chromosome. a, SMCX is transiently enriched on the inactive-X. Representative example (top) and quantification (bottom) of RNA FISH detection of Xist RNA (green) coupled with detection the Strep-SMCX (red) in $X X$ and $X^{\Delta T s i x} Y$ ESCs expressing mouse SMCX transgene $(T g-m S m c x)$; human Strep-SMCX transgene (Tg-hSMCX); Strep-SMCX mutant transgene lacking demethylase activity $(T g-h S M C X(H 514 A))$; and, Strep-SMCY transgene $(T g-h S M C Y) . \mathrm{n}=100$ nuclei from each of 3 independent ESC lines. b, H3K4me2 exclusion from the Xist RNA-coated X-chromosome. Left, schematic and observed micrographs. Right, quantification of the data. cd, H3K4me2 occupancy by ChIP-Seq with varying SMCX dose. Normalized H3K4me2 ChIP signals plotted over every $1 \mathrm{~kb}$ bin of the mouse genome (see Methods and Extended Data Fig. 7b-f). e, Normalized H3K4me2 ChIP-Seq read counts $\pm 1 \mathrm{~kb}$ of transcription start sites (TSSs) for the eight X-linked genes profiled by RNA FISH in Figs. 2a and 3f. The SMCX-dose dependent $\mathrm{H} 3 \mathrm{~K} 4 \mathrm{me} 2$ reduction was more evident in Lamp2, Mecp2, and Atrx, whose expression is also more sensitive to SMCX levels (Figs. 2a and 3f). Plots in c-e are from two independent differentiating EpiLC lines. 

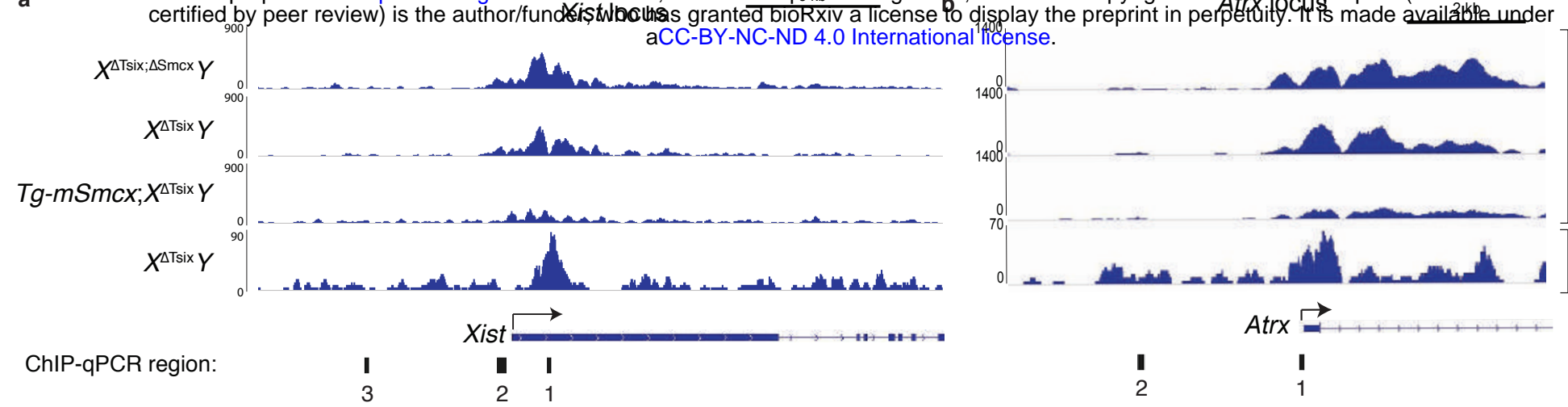
display the
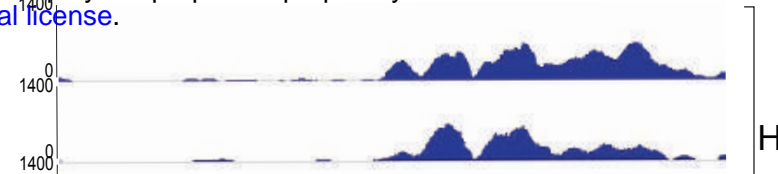

H3K4me2

c
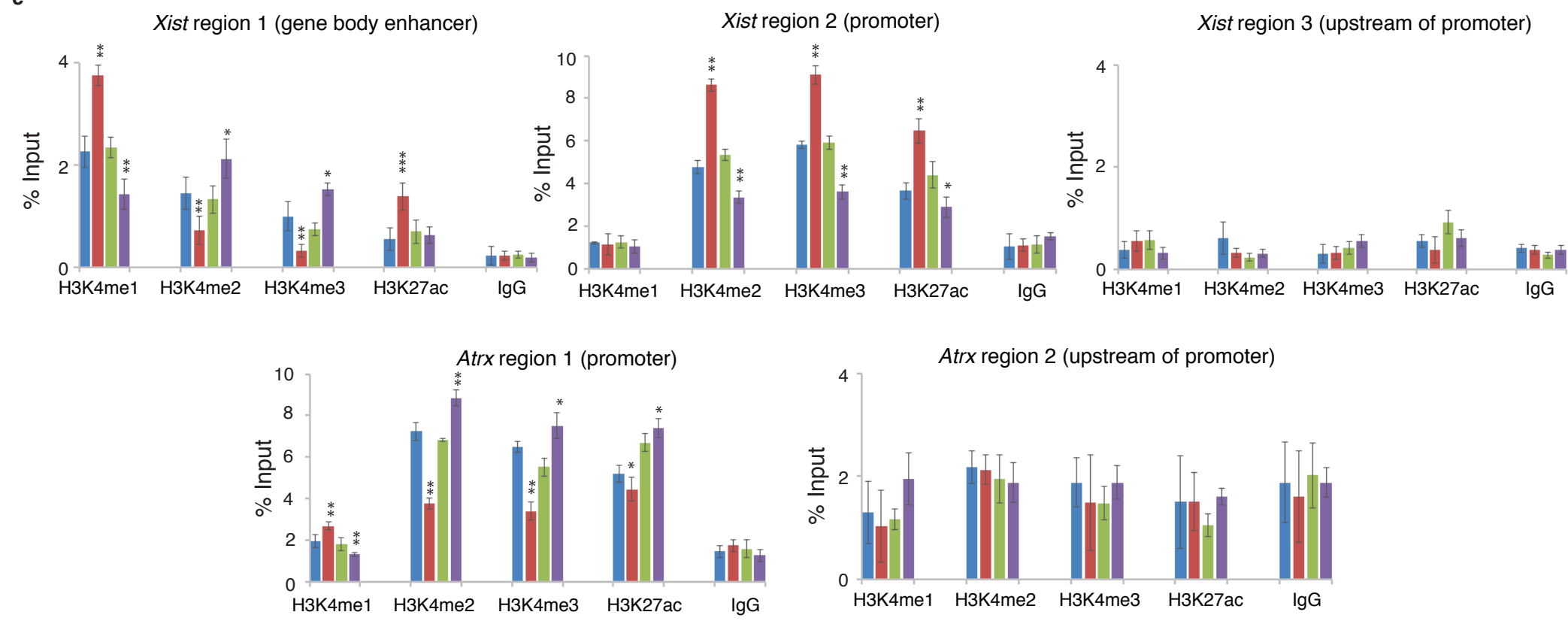

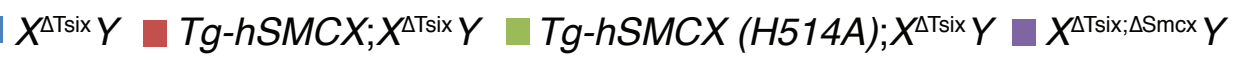

d

\begin{tabular}{l}
\hline S SMCX protein \\
$P$ H3K27me3 \& other modifications \\
\hline Polycomb group \& other proteins
\end{tabular}

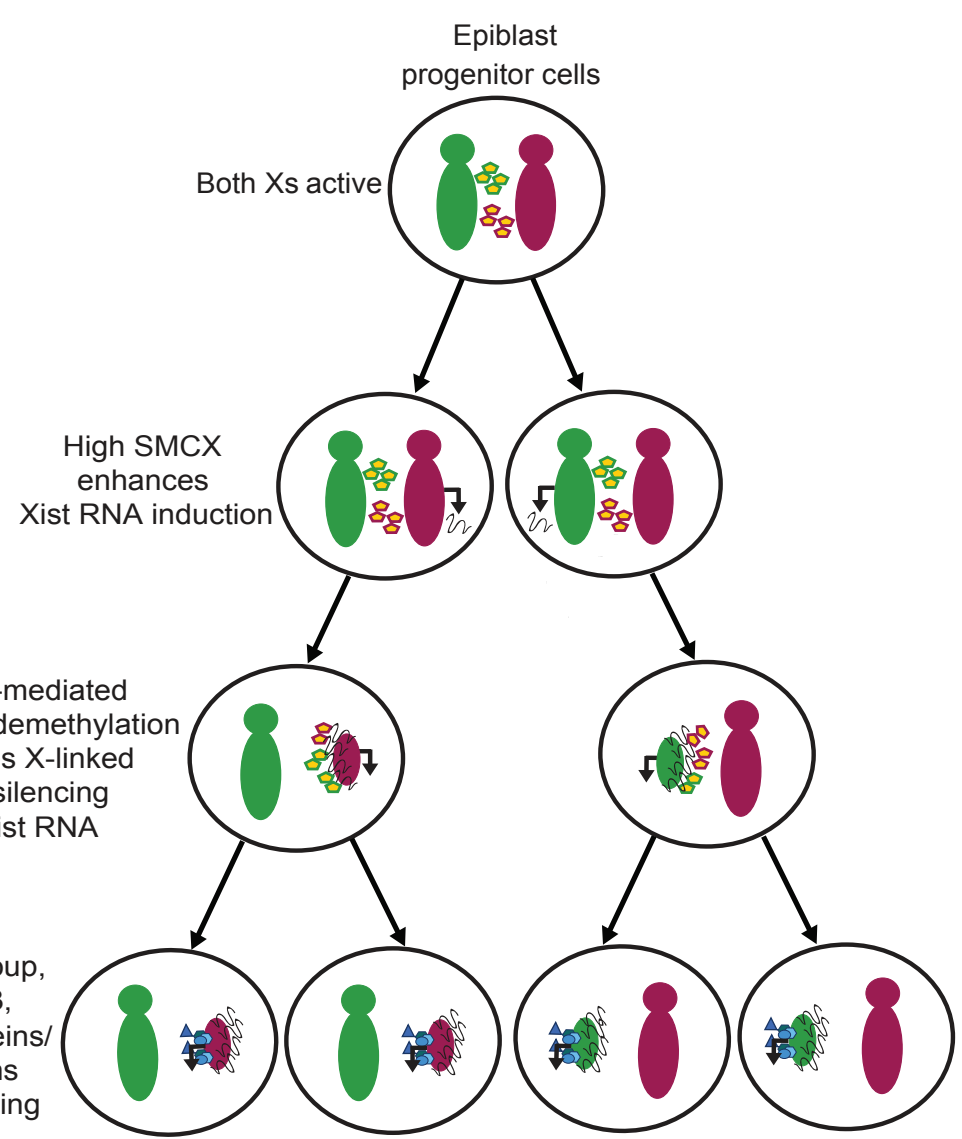


Figure 5: SMCX induces Xist expression via enhancer activation. a, SMCX and H3K4me2 occupancy at the Xist locus. H3K4me2 enrichment downstream of the Xist TSS is inversely correlated to SMCX dose and Xist expression. b, SMCX and H3K4me2 occupancy at the Atrx locus. c, ChIP-qPCR quantification of H3K4me1, H3K4me2, H3K4me3, and H3K4ac occupancy at the Xist and Atrx loci in d2 differentiated EpiLCs. Locations of PCR amplicons are denoted beneath the browser views in $\mathbf{a}$ and b. Mean \pm SEM. ${ }^{*}, p \leq 0.05 ; * *, p<0.01 ; * * *, p<$ 0.001, Welch's t-tests. d, A model of SMCX function in X-inactivation. 


\section{Methods}

Ethics Statement. This study was performed in strict accordance with the recommendations in the Guide for the Care and Use of Laboratory Animals of the National Institutes of Health. All animals were handled according to the protocols approved by the University Committee on Use and Care of Animals (UCUCA) at the University of Michigan (protocol \#PRO00004007).

Mice. The generation of $S m c x^{\mathrm{fl} / f \mathrm{l}}$ mice and mice harboring the $T \operatorname{six} \mathrm{AA}^{\mathrm{AA} \Delta 1.7}\left(T \operatorname{six}^{\Delta}\right)$ mutation have been described previously ${ }^{25,48}$. The X-linked GFP transgenic $(X-G F P)$ and JF1 strains have also been described previously $30,31,49,50$.

Embryo Dissections and Processing. Pre-, peri-, and post-implantation stage embryos were isolated essentially as described previously ${ }^{51}$. Briefly, E3.5 embryos were flushed from the uterine limbs in 1X PBS (Invitrogen, \#14200075) containing $6 \mathrm{mg} / \mathrm{ml}$ bovine serum albumin (BSA; Invitrogen, \#15260037). The zona pellucidas surrounding E3.5 embryos were removed through incubation in cold Acidic Tyrode's Solution (Sigma, \#T1788), followed by neutralization through several transfers of cold M2 medium (Sigma, \#M7167). GFP fluorescence conferred by the paternal transmission of the $X-G F P$ transgene was used to distinguish female from male embryos, since only females inherit the paternal X-chromosome. Isolated embryos were either lysed for RNA isolation or plated onto gelatin-coated glass coverslips in 1X PBS with $6 \mathrm{mg} / \mathrm{ml}$ BSA for immunofluorescence (IF)/RNA fluorescence in situ hybridization (FISH) staining. For plated embryos, excess solution was aspirated, and coverslips were air-dried for 15 min. After drying, embryos were permeabilized and fixed in $50 \mu \mathrm{L}$ solution of $0.05 \%$ Tergitol (Sigma, \#NP407) and 1\% paraformaldehyde (Electron Microscopy Sciences, \#15710) in 1X PBS for $10 \mathrm{~min}$. Excess solution was tapped off, and coverslips were rinsed $3 \mathrm{X}$ with $70 \%$ ethanol and stored in $70 \%$ ethanol at $-20^{\circ} \mathrm{C}$ prior to RNA FISH. For isolation of post-implantation embryos (E5.5, E6.5 and E7.5), individual implantation sites were cut from the uterine limbs and decidua were removed with forceps in $1 \mathrm{X}$ PBS $/ 6 \mathrm{mg} / \mathrm{ml} \mathrm{BSA}$. Embryos were dissected from the decidua, and the Reichert's membranes surrounding postimplantation embryos were removed using fine forceps. For separation of extra-embryonic and epiblast portions of embryos, fine forceps were used to physically bisect the embryos at the junction of the epiblast and extra-embryonic ectoderm. The epiblast was further distinguished by 
GFP fluorescence conferred by the paternally-transmitted $X$-GFP transgene; the transgene is mosaically expressed in the epiblast due to random $\mathrm{X}$-inactivation but is silenced in the extraembryonic tissues because of imprinted $X$-inactivation of the paternal- $X^{30,31,49,50}$. Extraembryonic and embryonic epiblast cells were then separately either lysed for RNA isolation or plated in $0.25 \mathrm{X}$ PBS with $6 \mathrm{mg} / \mathrm{mL}$ BSA onto gelatinized coverslips for IF/RNA FISH staining. Samples plated for IF/RNA FISH were fixed, permeabilized, and stored as described for E3.5 embryos.

Derivation, Culture and Differentiation of Embryonic Stem Cell (ESCs) Lines. ESC lines were derived following the protocol previously described ${ }^{52}$. Cells were cultured on mouse embryo fibroblasts (MEFs) in Knockout DMEM (GIBCO, \#10829-018) with 15\% Knockout Serum Replacement (GIBCO, \#A1099201), 5\% FBS (GIBCO, \#104390924), 2 mM L-glutamine (GIBCO, \#25030), 0.1 mM 2-mercaptoethanol (Sigma, \#M7522), 1 X nonessential amino acids (GIBCO, \#11140-050), supplemented with 1 MM GSK3 inhibitor CHIR99021 (Stemgent \#040004), $1 \mu \mathrm{M}$ MEK inhibitor PD0325901 (Stemgent \#04-0006) and 1000 units/mL LIF (Millipore

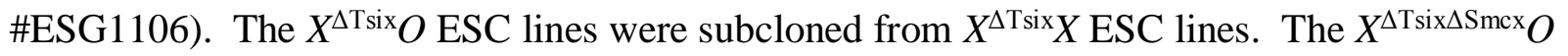
ESC lines were subcloned from $X^{\Delta \mathrm{Tsix} \Delta \mathrm{Smcx}} X$ ESC lines. The $X^{\Delta \mathrm{Smcx}} O$ and $X^{\mathrm{JF} 1} O$ ESC lines were subcloned from $X^{\mathrm{JF} 1} X^{\Delta \mathrm{Smcx}} \mathrm{ESC}$ line. The loss of the second $\mathrm{X}$-chromosome in the $X^{\Delta \mathrm{Smcx}} O$ and $X^{\mathrm{JF} 1} O$ ESC lines was ascertained via RNA and DNA FISH for Xist and the eight X-linked genes depicted in Fig. 2a.

For IF and/or RNA FISH, cells were then permeabilized through sequential treatment with ice-cold cytoskeletal extraction buffer (CSK:100 mM NaCl, $300 \mathrm{mM}$ sucrose, $3 \mathrm{mM}$ $\mathrm{MgCl}$, and $10 \mathrm{mM}$ PIPES buffer, pH 6. 8) for $30 \mathrm{sec}$, ice-cold CSK buffer containing $0.4 \%$ Triton X-100 (Fisher Scientific, \#EP151) for $30 \mathrm{sec}$, followed twice with ice-cold CSK for 30 sec each. After permeabilization, cells were fixed by incubation in $4 \%$ paraformaldehyde for 10 min. Cells were then rinsed $3 \mathrm{X}$ in $70 \%$ ethanol and stored in $70 \%$ ethanol at $-20^{\circ} \mathrm{C}$ prior to IF and/or RNA FISH.

Differentiation of ESCs into Epiblast-like Cells (EpiLCs). To convert ESC to EpiLC, ESC lines were grown in $2 \mathrm{i}$ culture conditions (N2B27 medium consisting 50\% DMEM/F12, 50\% neurobasal media, 2 mM L-glutamine (GIBCO, \#25030), 0.1 mM 2-mercaptoethanol (Sigma, 
\#M7522), N2 supplement (Invitrogen \#17502048), B27 supplement (Invitrogen \#17504-044), supplemented with $1 \mu$ M GSK3 inhibitor CHIR99021 (Stemgent \#04-0004), $1 \mu$ M MEK inhibitor PD0325901 (Stemgent \#04-0006), and 1000 U/ml LIF (Millipore \#ESG1106) in gelatin-coated tissue culture dishes for 4 passages (Buecker et al., 2014; Hayashi et al., 2011). To achieve EpiLC differentiation, cells were cultured in N2B27 medium supplemented with 10 ng/ml FGF2 (R\&D Systems, \#233-FB) and 20 ng/ml Activin A (R\&D Systems, \#338-AC) in Fibronectin $(15 \mu \mathrm{g} / \mathrm{ml})$ (Sigma \#F1141)-coated tissue culture dishes for 48 hrs. For further differentiation, the cells were cultured in N2B27 medium without FGF2 and Activin A for an additional 2, 4 and 6 days (d2, d4 and d6 of differentiation, respectively). For IF and/or RNA FISH, cells were permeabilized, fixed, and CSK processed as described above for ESCs.

Derivation and Culture of Epiblast Stem Cell (EpiSC) Lines. EpiSCs $\left(X^{\Delta \mathrm{Tsix}} X^{\mathrm{JF} 1} \# 2\right.$, \#15) were derived and characterized in a previous study ${ }^{33}$. For derivation of the EpiSCs lines, the epiblast layer was microdissected from E5.5 embryos and plated on MEF cells in EpiSC medium consisting of Knockout DMEM (GIBCO, \#10829-018) supplemented with 20\% Knockout Serum Replacement (KSR; GIBCO, \#A1099201), 2 mM L-glutamine (GIBCO, \#25030), 1X nonessential amino acids (GIBCO,\#11140-050), and 0.1 mM 2-mercaptoethanol (Sigma, \#M7522), 10 ng/ml FGF2 (R\&D Systems, \#233-FB) and cultured for 3-4 days to form a large EpiSC colony. EpiSC colonies were then manually dissociated into small clusters using a glass needle and plated into $1.9 \mathrm{~cm}^{2}$ wells containing MEF feeders in EpiSC cell medium. EpiSCs were passaged every third day using $1.5 \mathrm{mg} / \mathrm{ml}$ collagenase type IV (GIBCO, \#17104-019) with pipetting into small clumps.

RNA Fluorescence in situ Hybridization (RNA FISH). RNA FISH with double-stranded and strand-specific probes was performed as previously described ${ }^{14,49,53}$. The dsRNA FISH probes were made by randomly-priming DNA templates using BioPrime DNA Labeling System (Invitrogen, \#18094011). Strand-specific Xist probes were generated from templates as described $^{39,51}$. Probes were labeled with Fluorescein-12-dUTP or -UTP (Invitrogen) or Cy3$\mathrm{dCTP}$ or - CTP (GE Healthcare). Labeled probes from multiple templates were precipitated in a 0.3M sodium acetate solution (Teknova, \#S0298) along with $300 \mu \mathrm{g}$ of yeast tRNA (Invitrogen, \#15401-029) and $150 \mu \mathrm{g}$ of sheared, boiled salmon sperm DNA (Invitrogen, \#15632-011). The 
solution was then spun at $15,000 \mathrm{rpm}$ for $20 \mathrm{~min}$ at $4^{\circ} \mathrm{C}$. The pellet was washed consecutively with $70 \%$ ethanol and 100\% ethanol. The pellet was dried and re-suspended in deionized formamide (ISC Bioexpress, \#0606). The probe was denatured by incubating at $90^{\circ} \mathrm{C}$ for $10 \mathrm{~min}$ followed by an immediate 5 min incubation on ice. A $2 X$ hybridization solution consisting of 4X SSC, 20\% Dextran sulfate (Millipore, \#S4030), and $2.5 \mathrm{mg} / \mathrm{ml}$ purified BSA (New England Biolabs, \#B9001S) was added to the denatured solution. All probes were stored at $-20^{\circ} \mathrm{C}$ until use.

Cells or embryo fragments mounted on coverslips were dehydrated through $2 \mathrm{~min}$ incubations in $70 \%, 85 \%, 95 \%$, and $100 \%$ ethanol solutions and subsequently air-dried. The coverslips were then hybridized to the probe overnight in a humid chamber at $37^{\circ} \mathrm{C}$. The samples were then washed $3 \mathrm{X}$ for $7 \mathrm{~min}$ each while shaking at $39^{\circ} \mathrm{C}$ with $2 \mathrm{XSSC} / 50 \%$ formamide, 2X with 2X SSC, and 2X with 1X SSC. A 1:250,000 dilution of DAPI (Invitrogen, \#D21490) was added to the third 2X SSC wash. Coverslips were then mounted on slides in Vectashield (Vector Labs, \#H-1200).

Allele-specific Xist RNA FISH. Allele specific Xist RNA FISH were generated as described by Levesque et al. (2013) ${ }^{54}$. Briefly, a panel of short oligonucleotide probes were designed to uniquely detect either the M. musculus or the M. molossinus alleles of Xist. Five probes were designed for each Xist allele; each probe overlaps a SNP that differs between the two strains, with the SNP located at the fifth base pair position from the 5' end. The same panel of five SNPs was used for both sets of allele-specific probes. The 3' end of each oligonucleotide probe is fluorescently tagged using Quasar dyes (Biosearch technologies): M. musculus-specific oligos were labeled with Quasar 570 and M. molossinus oligos labeled with Quasar 670. In addition to labeled SNP-overlapping oligonucleotides, a panel of 5 "mask" oligonucleotides was also synthesized. These "mask" probes are complimentary to the 3' end of the labeled allele-specific probes and will initially hybridize to the allele-specific oligonucleotides, leaving only 9-10 base pairs of sequence surrounding the polymorphic site available to initially hybridize to the target Xist RNA; since this region is short, the presence of a single nucleotide polymorphism is sufficient to destabilize unintended hybridization with the alternate allele. Sequences of detection and mask probes are listed in Methods Table 1 below. The allele-specific Xist RNA FISH probes were tested together with a strand-specific Xist RNA probe, labeled with 
Fluorescein-12-UTP (Invitrogen), which served as a guide probe that hybridized to Xist RNA generated from both Xist alleles and ensured that the allele-specific probes were faithfully detecting Xist RNA. The guide Xist RNA probe was first ethanol precipitated as previously described, then resuspended in hybridization buffer containing $10 \%$ dextran sulfate, $2 \mathrm{X}$ salinesodium citrate (SSC) and 10\% formamide. The precipitated guide RNA probe was then mixed with the M. musculus and M. molossinus detection probes, to a final concentration of $5 \mathrm{nM}$ per allele-specific oligo, and $10 \mathrm{nM}$ mask probe, yielding to 1:1 mask:detection oligonucleotide ratios. Coverslips were hybridized to the combined probe overnight in a humid chamber at $37^{\circ} \mathrm{C}$. After overnight hybridization, samples were washed twice in $2 \mathrm{X}$ SSC with $10 \%$ formamide at $37^{\circ} \mathrm{C}$ for $30 \mathrm{~min}$, followed by two washes in $2 \mathrm{X} \mathrm{SSC}$ for 5 mins. A 1:250,000 dilution of DAPI (Invitrogen, \#D21490) was added to the second 2X SSC wash. Coverslips were then mounted on slides in Vectashield (Vector Labs, \#H-1200).

DNA FISH. DNA FISH probes were prepared as described for double-stranded RNA-FISH probes $^{14,53}$. The BAC template used for Xist DNA FISH is RP24-287F13 (Children's Hospital of Oakland Research Institute). After RNA FISH, cells were washed with 1X PBS three times and then incubated in PBS for $5 \mathrm{~min}$ at room temperature. The cells were then refixed with $1 \%$ (wt/vol) PFA containing 0.5\% (vol/vol) Tergitol and 0.5\% (vol/vol) Triton X-100 for $10 \mathrm{~min}$ at room temperature. The cells were next dehydrated through an ethanol series $(70 \%, 85 \%$, and $100 \%$ ethanol, 2 min each) and air dried for 15 mins. The cells were then treated with RNase A $(1.25 \mu \mathrm{g} / \mu \mathrm{l})$ at $37^{\circ} \mathrm{C}$ for $30 \mathrm{~min}$. The cells were again dehydrated through the ethanol series as described above. The samples were then denatured in a prewarmed solution of $70 \%$ formamide in $2 \mathrm{X} \mathrm{SSC}$ on a glass slide stationed on top of a heat block set at $95^{\circ} \mathrm{C}$ for $11 \mathrm{~min}$ followed immediately by dehydration through a $-20^{\circ} \mathrm{C}$-chilled ethanol series $(70 \%, 85 \%, 95 \%$, and $100 \%$ ethanol, $2 \mathrm{~min}$ each). The cells were then air dried for $15 \mathrm{~min}$ followed by probe hybridization overnight at $37^{\circ} \mathrm{C}$. The following day, the samples were washed twice with prewarmed $50 \%$ formamide/2X SSC solution at $39^{\circ} \mathrm{C}$ and $2 \mathrm{X}$ with $2 \mathrm{X} \mathrm{SSC,} 7 \mathrm{~min}$ each.

Immunofluorescence (IF). Cells mounted on glass coverslips were washed 3X in PBS for 3 min each while shaking. Coverslips were then incubated in blocking buffer consisting of 0.5 mg/mL BSA (New England Biolabs, \#B9001S), 50 g/mL yeast tRNA (Invitrogen, \#15401029), 80 units/mL RNAseOUT (Invitrogen, \#10777-019), and 0.2\% Tween 20 (Fisher, \#BP337- 
$100)$ in $1 \mathrm{X}$ PBS in a humid chamber for $30 \mathrm{~min}$ at $37^{\circ} \mathrm{C}$. The samples were next incubated with primary antibody diluted in blocking buffer for $1-3 \mathrm{hr}$ in the humid chamber at $37^{\circ} \mathrm{C}$. AntiREX1 antibody (Thermo Scientific, \#PA5-27567) was used at 1:150 dilution; anti-HA antibody was used at 1:100 dilution (Abcam, \#18181); anti-H3K4me2 was used at 1:200 (Millipore, \#07030); and, the anti-H3S10ph antibody (Cell Signaling, \#9701) was used at 1:150 dilution. The Cleaved Caspase-3 (ASP175) (5A1E) antibody (Cell Signaling \# 9664) was used at 1:200. The samples were then washed $3 \mathrm{X}$ in PBS/0.2\% Tween 20 for 3 min each while shaking. After a 5 min incubation in blocking buffer at $37^{\circ} \mathrm{C}$ in the humid chamber, the samples were incubated in blocking buffer containing a 1:300 dilution of fluorescently-conjugated secondary antibody (Alexa Fluor, Invitrogen) for $30 \mathrm{~min}$ in the humid chamber at $37^{\circ} \mathrm{C}$, followed by three washes in PBS/0.2\% Tween 20 while shaking for 3 min each. The samples were then processed for RNA FISH.

RT-qPCR. RT-qPCR was performed using SYBR Green-based relative quantification method on an Eppendorf Realplex Mastercycler. The housekeeping gene Gapdh was used as an internal control for data normalization. cDNA was synthesized using SuperScript III First Strand Synthesis System (Invitrogen \# 18080-051), following manufacturer's instructions. Control reactions lacking reverse transcriptase for each sample were also performed to rule out genomic DNA contamination. Real time PCR was carried out using the SYBR Green PCR Mastermix (Kapa Biosystem \#KK4650). The following primers were used for RT and PCR: Smcx: forward TTTGGCAGCGGTTTCCCTGTCAGT, reverse AAGACCATTCCCACATACAGCC; Rexl: forward CCACAAACAGATCCGGCTT, reverse TGGAAGCGAGTTCCCTTCTC; Xist: forward CAAGAAGAAGGATTGCCTGGATTT, reverse GCGAGGACTTGAAGAGAAGTTCTG. $P$-values for all RT-qPCR results were calculated using Welch's two-sample T-tests.

Quantification of Allele-specific Expression by Pyrosequencing. Allele-specific expression of Smcx was quantified using Qiagen PyroMark sequencing platform. An Smcx RT-PCR amplicon containing a single nucleotide polymorphism (SNP) was designed using PyroMark Assay Design software. Single cell lysates were prepared in $10 \mu \mathrm{L}$ of lysis buffer from the Single Cell RT-PCR Assay Kit by Signosis (\# CL-0002). DNA was removed from samples by spinning at $12000 \mathrm{rpm}$ for $5 \mathrm{~min}$, followed by DNase I treatment $\left(37^{\circ} \mathrm{C}\right.$ for $\left.30 \mathrm{~min}\right)$. Five $\mu \mathrm{l}$ of 
cell lysate was then used directly for cDNA synthesis with the Invitrogen SuperScript III OneStep RT-PCR System (\#12574-026). Control reactions lacking reverse transcriptase for each sample were also performed to rule out genomic DNA contamination. Following RT-PCR, $5 \mu 1$ of each $25 \mu \mathrm{L}$ reaction was run on a $3 \%$ agarose gel to assess the efficacy of the reverse transcription and amplification. The samples were then prepared for Pyrosequencing according to the standard recommendations for use with the PyroMark Q96 ID sequencer. The following primers were used for Smcx RT-PCR and Pyrosequencing: forward AGTCCAGGGCTGCTGCAGT; reverse, 5' -biotin GGAGCCAGCGTGCGTTTTA; sequencing, CAGGTGGAACAGGCG .

\section{Expression Plasmids and Lentiviral Transduction in ESCs. $m S m c x, h S M C X$, and $h S M C Y$} were cloned from cDNA libraries into the Gateway Entry vector (pENTR/D-TOPO, Invitrogen). The SMCX mutant H514A cDNA was generated by PCR-based mutagenesis. The sequence of all cDNAs was verified via Sanger sequencing. To generate the plasmids for stable overexpression in $X^{\Delta T s i x} Y$ male ESCs, the cDNAs were cloned into an in-house generated $P G K$ promoter-driven Strep-tag II lentiviral vector by Gateway LR recombination. To generate doxycycline inducible constructs used in Fig. 3A-B, the cDNAs were cloned into a $P G K$ promoter-driven HA-tag lentiviral vector pLIX-402 (Addgene plasmid \# 41394) by Gateway LR recombination. For production of lentivirus, 50-70\% confluent HEK293T cells in a 10-cm tissue culture plate were co-transfected with $3.33 \mu \mathrm{g}$ lentiviral construct, $2.5 \mu \mathrm{g}$ psPAX2 packaging plasmid and $1 \mu \mathrm{g}$ pMD2.G envelope plasmid using TransIT-293 (Mirus). Lentiviral particles were collected after 48 hrs of post transfection. The lentivral particles were then concentrated using LentiX (Clontech \#631231). Concentrated lentivirus was transduced to ESCs with 10ug/ml Polybrene (Millipore, \#TR-1003-G). After 72 hrs, the transduced cells were selected with 3ug/ml Puromycin (Sigma, \#P8833-25MG) and subcloned to generate clonal cell lines. For inducible overexpression, cells were treated with $2 \mathrm{ug} / \mathrm{ml}$ doxycycline (Sigma) for 3 days. Media were changed every 24 hours with fresh doxycycline.

ChIP-Seq. ChIP was performed as previously described ${ }^{55}$, with a minor modification, where we sonicated the chromatin using truChIP sonicator (Covaris). Two independent $X^{\Delta \mathrm{Tsix}} Y$, $X^{\Delta \mathrm{Tsix} ; \Delta \mathrm{Smcx}} Y$, or $T g-m S m c x ; X^{\Delta \mathrm{Tsix}} Y$ ESC lines were differentiated into EpiLCs for all ChIP-Seq 
experiments with the exception that SMCX ChIP-Seq was performed on one $X^{\Delta \mathrm{Tsix} ; \Delta \mathrm{Smcx}} Y$ EpiLCs sample. To achieve optimal inter-sample normalization of ChIP efficiency, Drosophila chromatin, which contains fly-specific histone variant $\mathrm{H} 2 \mathrm{Av}$, was spiked in and subsequently immunoprecipitated with an anti-H2Av antibody (Active Motif) according to the manufacturers instruction. Two to five million EpiLCs were lysed and $3 \mu \mathrm{g}$ of H3K4me2 antibody (Abcam \#7766) or $10 \mu \mathrm{g}$ of an in-house anti-SMCX antibody ${ }^{55}$ was used in individual ChIP reactions. ChIP-Seq libraries were sequenced on the Illumina HiSeq2500 platform to generate $50 \mathrm{bp}$ singleend reads. Raw reads were demultiplexed and filtered according to the standard Illumina analysis pipeline. Reads from sequencing libraries were then mapped to the mouse (mm9) and fruit fly (dm6) genome assemblies using Bowtie ${ }^{56}$ allowing up to 2 mismatches. Only uniquelymapped reads were used for analysis. Replicate BAM files were merged and reads were extended by $180 \mathrm{bp}$ and converted to BED files. Basic sequencing statistics of all samples are presented in Methods Table 2.

For H3K4me2 analysis, Drosophila-normalized read coverage across the different genotypes was calculated over 1-kb bins across the mouse genome. Each 1-kb bin was normalized to the merged input samples. For SMCX, peaks were called using MACS2 software (version 2.1.0.20140616) ${ }^{57}$ using input bam files for normalization, with filters for a q-value < 0.1 and a fold enrichment greater than 1 . This yielded 98 peaks on the X-chromosome for $X^{\Delta \mathrm{Tsix}} Y$ samples and only 1 peak for the $X^{\Delta \mathrm{Tsix} ; \Delta \mathrm{Smcx} Y}$ sample. DNase I hypersensitive sites (DHS) were defined using MACS2 $(q<0.05)$ from the ENCODE mouse ESC data $\operatorname{set}^{58}$. Promoters were defined as $\pm 1 \mathrm{~kb}$ from annotated transcription start sites (TSS) of the mm9 assembly. To find SMCX peaks, and subsequently H3K4me2 density, at DHS or promoters, we used the Bedtools intersect command to select SMCX peaks in which 10\% of the SMCX peak length overlapped the promoters or DHS. For visualization in the Integrated Genome Viewer, bigwig files were generated with coverage normalized using the number of mapped reads to the Drosophila genome (reads mapped per reference genome per million reads) ${ }^{59,60}$.

ChIP-qPCR. ChIP was performed as above. Two to five million cells were lysed and $3 \mu \mathrm{g}$ of H3K4me1 antibody (Abcam \#8895) or $3 \mu \mathrm{g}$ of H3K4me2 antibody (Abcam \#7766) or $3 \mu \mathrm{g}$ of H3K4me3 antibody (Abcam \#8580) or $3 \mu \mathrm{g}$ of H3K27ac antibody (Active motif \#39135) or $3 \mu \mathrm{g}$ of rabbit IgG antibody (Jackson Immuno Research \#011-000-003) was used in individual ChIP 
reactions. DNA was purified by phenol/chloroform extraction and eluted using QIAquick purification kit (QIAGEN\#28104). All qPCR reactions were performed using SYBR Greenbased relative quantification method on an Eppendorf Realplex Mastercycler.

Fluorescent Western blotting. The Odyssey CLx system (LI-COR Biosciences, Lincoln, NE, USA) was used for fluorescent Western blotting. Protein lysates were made in RIPA buffer (50mM Tris-HCl, $1 \%$ NP40, 0.25\% Na-deoxycholate, 150mM NaCl) with PMSF (Sigma, \#P7626) and protease inhibitor cocktail (Roche, \#11873580001). Lysates were mixed with Laemmli sample buffer and boiled for $10 \mathrm{~min}$ prior to loading on Polyacrylamide gels. Resolved proteins were transferred onto PVDF membranes (Immobilon-P, Millipore) overnight.

Membranes were then blocked in 5\% BSA (Sigma, \#A7906) for $1 \mathrm{hr}$ at room temperature, and incubated in the anti-Strep-Tag II (GenScript, \#A01732-100) and anti-Actin (Sigma, \#A5060) primary antibodies together overnight at $4^{\circ} \mathrm{C}$. Appropriate Li-COR IRDye series (680LT/800LT) secondary antibodies were used. Blots were scanned using the Odyssey CLx Imager (Li-COR Biosciences) following the manufacturer's instruction. Results were analyzed using Odyssey Li-COR software. Transgenic SMCX levels were normalized to ACTIN in each blot.

Microscopy. Images of all stained samples were captured using a Nikon Eclipse TiE inverted microscope with a Photometrics CCD camera. The images were analyzed after deconvolution using NIS-Elements software. All images were processed uniformly.

\section{SMCX/SMCY Sequence Analysis.}

Amino acid sequences were obtained from the Ensembl database and from Cortez et al., $2014^{61}$. Multiple sequence alignment was performed using MSAViewer ${ }^{62}$. MSAViewer tree tool was used to create a rooted phylogenetic tree with branch lengths according to the Neighbor Joining algorithm. Finally, the alignment was analyzed to find amino acids conserved across mammalian (human, mouse, and elephant) SMCX, but not in mammalian SMCY sequences nor in the orthologous chicken KDM5A sequence. These sites were visualized within the domain structure of human SMCX using PROSITE (http://prosite.expasy.org/), an available ExPASy bioinformatic resource tool. 


\section{REFERENCES}

1 Ohno, S., Kaplan, W. D. \& Kinosita, R. Formation of the sex chromatin by a single Xchromosome in liver cells of Rattus norvegicus. Exp Cell Res 18, 415-418 (1959).

2 Ohno, S. Sex chromosomes and sex-linked genes. (Springer-Verlag, 1967).

3 Lyon, M. F. Gene action in the X-chromosome of the mouse (Mus musculus L.). Nature 190, 372-373 (1961).

4 Marahrens, Y., Panning, B., Dausman, J., Strauss, W. \& Jaenisch, R. Xist-deficient mice are defective in dosage compensation but not spermatogenesis. Genes Dev 11, 156-166 (1997).

5 Penny, G. D., Kay, G. F., Sheardown, S. A., Rastan, S. \& Brockdorff, N. Requirement for Xist in X chromosome inactivation. Nature 379, 131-137 (1996).

6 Brown, C. J. et al. A gene from the region of the human X inactivation centre is expressed exclusively from the inactive X chromosome. Nature 349, 38-44 (1991).

7 Sheardown, S. A. et al. Stabilization of Xist RNA mediates initiation of X chromosome inactivation. Cell 91, 99-107 (1997).

8 Kay, G. F. et al. Expression of Xist during mouse development suggests a role in the initiation of X chromosome inactivation. Cell 72, 171-182 (1993).

9 Clemson, C. M., McNeil, J. A., Willard, H. F. \& Lawrence, J. B. XIST RNA paints the inactive $\mathrm{X}$ chromosome at interphase: evidence for a novel RNA involved in nuclear/chromosome structure. J Cell Biol 132, 259-275 (1996).

10 Brown, C. J. et al. The human XIST gene: analysis of a $17 \mathrm{~kb}$ inactive X-specific RNA that contains conserved repeats and is highly localized within the nucleus. Cell 71, 527542 (1992).

11 McHugh, C. A. et al. The Xist lncRNA interacts directly with SHARP to silence transcription through HDAC3. Nature, doi:10.1038/nature14443 (2015).

12 Chu, C. et al. Systematic discovery of xist RNA binding proteins. Cell 161, 404-416, doi:10.1016/j.cell.2015.03.025 (2015).

13 Minajigi, A. et al. Chromosomes. A comprehensive Xist interactome reveals cohesin repulsion and an RNA-directed chromosome conformation. Science 349, doi:10.1126/science.aab2276 (2015).

14 Gayen, S., Maclary, E., Hinten, M. \& Kalantry, S. Sex-specific silencing of X-linked genes by Xist RNA. Proc Natl Acad Sci U S A 113, E309-318, doi:10.1073/pnas.1515971113 (2016). 
15 Iwase, S. et al. The X-linked mental retardation gene SMCX/JARID1C defines a family of histone H3 lysine 4 demethylases. Cell 128, 1077-1088, doi:10.1016/j.cell.2007.02.017 (2007).

16 Tahiliani, M. et al. The histone H3K4 demethylase SMCX links REST target genes to Xlinked mental retardation. Nature 447, 601-605, doi:10.1038/nature05823 (2007).

17 Barski, A. et al. High-resolution profiling of histone methylations in the human genome. Cell 129, 823-837, doi:10.1016/j.cell.2007.05.009 (2007).

18 Kouzarides, T. Chromatin modifications and their function. Cell 128, 693-705, doi:S0092-8674(07)00184-5 [pii]

10.1016/j.cell.2007.02.005 (2007).

19 Sun, B. K., Deaton, A. M. \& Lee, J. T. A transient heterochromatic state in Xist preempts $\mathrm{X}$ inactivation choice without RNA stabilization. Mol Cell 21, 617-628, doi:S10972765(06)00050-5 [pii]

10.1016/j.molcel.2006.01.028 (2006).

20 Boggs, B. A. et al. Differentially methylated forms of histone H3 show unique association patterns with inactive human X chromosomes. Nat Genet 30, 73-76 (2002).

21 Carrel, L., Hunt, P. A. \& Willard, H. F. Tissue and lineage-specific variation in inactive $\mathrm{X}$ chromosome expression of the murine Smcx gene. Hum Mol Genet 5, 1361-1366 (1996).

22 Sheardown, S., Norris, D., Fisher, A. \& Brockdorff, N. The mouse Smcx gene exhibits developmental and tissue specific variation in degree of escape from X inactivation. Hum Mol Genet 5, 1355-1360 (1996).

23 Agulnik, A. I. et al. A novel X gene with a widely transcribed Y-linked homologue escapes X-inactivation in mouse and human. Hum Mol Genet 3, 879-884 (1994).

24 Wu, J. et al. Isolation and characterization of XE169, a novel human gene that escapes Xinactivation. Hum Mol Genet 3, 153-160 (1994).

25 Iwase, S. et al. A Mouse Model of X-linked Intellectual Disability Associated with Impaired Removal of Histone Methylation. Cell reports 14, 1000-1009, doi:10.1016/j.celrep.2015.12.091 (2016).

26 Lewandoski, M., Wassarman, K. M. \& Martin, G. R. Zp3-cre, a transgenic mouse line for the activation or inactivation of loxP-flanked target genes specifically in the female germ line. Curr Biol 7, 148-151, doi:S0960-9822(06)00059-5 [pii] (1997).

27 Takagi, N., Wake, N. \& Sasaki, M. Cytologic evidence for preferential inactivation of the paternally derived X chromosome in XX mouse blastocysts. Cytogenet Cell Genet 20, 240-248 (1978). 
28 Mak, W. et al. Reactivation of the paternal X chromosome in early mouse embryos. Science 303, 666-669 (2004).

29 Kay, G. F., Barton, S. C., Surani, M. A. \& Rastan, S. Imprinting and X chromosome counting mechanisms determine Xist expression in early mouse development. Cell 77, 639-650 (1994).

30 Hadjantonakis, A. K., Gertsenstein, M., Ikawa, M., Okabe, M. \& Nagy, A. Non-invasive sexing of preimplantation stage mammalian embryos. Nat Genet 19, 220-222 (1998).

31 Kalantry, S. \& Magnuson, T. The Polycomb group protein EED is dispensable for the initiation of random X-chromosome inactivation. PLoS Genet 2, e66, doi:10.1371/journal.pgen.0020066 (2006).

32 McMahon, A., Fosten, M. \& Monk, M. X-chromosome inactivation mosaicism in the three germ layers and the germ line of the mouse embryo. J Embryol Exp Morphol 74, 207-220 (1983).

33 Gayen, S., Maclary, E., Buttigieg, E., Hinten, M. \& Kalantry, S. A Primary Role for the Tsix lncRNA in Maintaining Random X-Chromosome Inactivation. Cell reports 11, 1251-1265, doi:10.1016/j.celrep.2015.04.039 (2015).

34 Gontan, C. et al. RNF12 initiates X-chromosome inactivation by targeting REX1 for degradation. Nature 485, 386-390, doi:nature11070 [pii]

10.1038/nature11070 (2012).

35 Hayashi, K., Ohta, H., Kurimoto, K., Aramaki, S. \& Saitou, M. Reconstitution of the mouse germ cell specification pathway in culture by pluripotent stem cells. Cell 146, 519-532, doi:10.1016/j.cell.2011.06.052 (2011).

36 Marahrens, Y., Loring, J. \& Jaenisch, R. Role of the Xist gene in X chromosome choosing. Cell 92, 657-664 (1998).

37 Gayen, S., Maclary, E., Hinten, M. \& Kalantry, S. Sex-specific silencing of X-linked genes by the Xist RNA. Proc Natl Acad Sci U S A (In press) (2016).

38 Allis, C. D. et al. New nomenclature for chromatin-modifying enzymes. Cell 131, 633636, doi:10.1016/j.cell.2007.10.039 (2007).

39 Sarkar, M. K. et al. An Xist-activating antisense RNA required for X-chromosome inactivation. Nat Commun 6, 8564, doi:10.1038/ncomms9564 (2015).

40 Heintzman, N. D. et al. Distinct and predictive chromatin signatures of transcriptional promoters and enhancers in the human genome. Nat Genet 39, 311-318, doi:10.1038/ng1966 (2007). 
41 Heintzman, N. D. et al. Histone modifications at human enhancers reflect global celltype-specific gene expression. Nature 459, 108-112, doi:10.1038/nature07829 (2009).

42 Rada-Iglesias, A. et al. A unique chromatin signature uncovers early developmental enhancers in humans. Nature 470, 279-283, doi:10.1038/nature09692 (2011).

43 Avner, P. \& Heard, E. X-chromosome inactivation: counting, choice and initiation. Nat Rev Genet 2, 59-67, doi:10.1038/35047580 (2001).

44 Augui, S., Nora, E. P. \& Heard, E. Regulation of X-chromosome inactivation by the Xinactivation centre. Nat Rev Genet 12, 429-442, doi:10.1038/nrg2987 (2011).

45 Lahn, B. T. \& Page, D. C. Four evolutionary strata on the human X chromosome. Science 286, 964-967, doi:7960 [pii] (1999).

46 Jegalian, K. \& Page, D. C. A proposed path by which genes common to mammalian X and Y chromosomes evolve to become X inactivated. Nature 394, 776-780, doi:10.1038/29522 (1998).

47 Masui, S. et al. Rex1/Zfp42 is dispensable for pluripotency in mouse ES cells. BMC Dev Biol 8, 45, doi:10.1186/1471-213X-8-45 (2008).

48 Sado, T., Wang, Z., Sasaki, H. \& Li, E. Regulation of imprinted X-chromosome inactivation in mice by Tsix. Development 128, 1275-1286 (2001).

49 Kalantry, S., Purushothaman, S., Bowen, R. B., Starmer, J. \& Magnuson, T. Evidence of Xist RNA-independent initiation of mouse imprinted X-chromosome inactivation. Nature 460, 647-651, doi:nature08161 [pii]

10.1038/nature08161 (2009).

50 Kalantry, S. et al. The Polycomb group protein Eed protects the inactive X-chromosome from differentiation-induced reactivation. Nat Cell Biol 8, 195-202, doi:ncb1351 [pii]

10.1038/ncb1351 (2006).

51 Maclary, E. et al. Differentiation-dependent requirement of Tsix long non-coding RNA in imprinted X-chromosome inactivation. Nat Commun 5, 4209, doi:10.1038/ncomms5209 (2014).

52 Bryja, V., Bonilla, S. \& Arenas, E. Derivation of mouse embryonic stem cells. Nat Protoc 1, 2082-2087, doi:10.1038/nprot.2006.355 (2006).

53 Hinten, M., Maclary, E., Gayen, S., Harris, C. \& Kalantry, S. Visualizing Long Noncoding RNAs on Chromatin. Methods Mol Biol 1402, 147-164, doi:10.1007/978-14939-3378-5_12(2016). 
54 Levesque, M. J., Ginart, P., Wei, Y. \& Raj, A. Visualizing SNVs to quantify allelespecific expression in single cells. Nature methods 10, 865-867, doi:10.1038/nmeth.2589 (2013).

55 Iwase, S. et al. A Mouse Model of X-linked Intellectual Disability Associated with Impaired Removal of Histone Methylation. Cell reports, doi:10.1016/j.celrep.2015.12.091 (2016).

56 Langmead, B., Trapnell, C., Pop, M. \& Salzberg, S. L. Ultrafast and memory-efficient alignment of short DNA sequences to the human genome. Genome biology 10, R25, doi:10.1186/gb-2009-10-3-r25 (2009).

57 Zhang, Y. et al. Model-based analysis of ChIP-Seq (MACS). Genome biology 9, R137, doi:10.1186/gb-2008-9-9-r137 (2008).

58 Yue, F. et al. A comparative encyclopedia of DNA elements in the mouse genome. Nature 515, 355-364, doi:10.1038/nature13992 (2014).

59 Orlando, D. A. et al. Quantitative ChIP-Seq normalization reveals global modulation of the epigenome. Cell reports 9, 1163-1170, doi:10.1016/j.celrep.2014.10.018 (2014).

60 Egan, B. et al. An Alternative Approach to ChIP-Seq Normalization Enables Detection of Genome-Wide Changes in Histone H3 Lysine 27 Trimethylation upon EZH2 Inhibition. PLoS One 11, e0166438, doi:10.1371/journal.pone.0166438 (2016).

61 Cortez, D. et al. Origins and functional evolution of Y chromosomes across mammals. Nature 508, 488-493, doi:10.1038/nature13151 (2014).

62 Yachdav, G. et al. MSAViewer: interactive JavaScript visualization of multiple sequence alignments. Bioinformatics 32, 3501-3503, doi:10.1093/bioinformatics/btw474 (2016). 


\section{Acknowledgments}

We thank members of the Kalantry and Iwase laboratories for discussions and critical review of the manuscript; Jacob Mueller for critically evaluating the manuscript; Angela Andersen of Pickersgill and Andersen, Life Science Editors (lifescienceeditors.com/), for editing services. We thank Michael Hinten for designing the Smcx Pyrosequencing assay; and, Paul Ginart and Arjun Raj for help with designing of the allele-specific Xist RNA FISH assay; and Saurabh Agarwal for technical assistance for genomic analyses. We acknowledge the services of the University of Michigan Sequencing Core Facility, supported in part by the University of Michigan Comprehensive Cancer Center. This work was funded by NIH National Research Service Awards 5-T32-GM07544 (University of Michigan Predoctoral Genetics Training Program; to E.M. and C.N.V.), T32-HD079342 (University of Michigan Predoctoral Career

Training in the Reproductive Sciences Program; to E.M., C.N.V., and R.S.P), 1F31HD080280-

01 (to E.M.), and a National Science Foundation Graduate Research Fellowship DGE-1256360 (to P.M.G.), a Rackham Predoctoral Fellowship from the University of Michigan (to E.M.), an NIH Director's New Innovator Award (DP2-OD-008646) (to S.K.), a March of Dimes Basil O’Connor Starter Scholar Research Award (5-FY12-119) (to S.K.), NIH NINDS Award (R01NS089896) (to S.I.), Farrehi Family Foundation Grant (to S.I.), a Reproductive Science Program Pilot Grant (to S.K. and S.I.), and the University of Michigan Endowment for Basic Sciences (S.K. and S.I.). Sequencing data generated for this study have been submitted to the NCBI Gene Expression Omnibus (GEO; http://www.ncbi.nlm.nih.gov/geo/) under accession number GSE96740. 


\section{Author Contributions}

S.G., S.I., and S.K. conceived the study and designed the experiments. S.I. generated

$S m c x^{\mathrm{fl}}$ mouse strain. S.G. bred Smcx mice, derived, characterized, transduced, and analyzed ESC, EpiLC, and EpiSC lines. E.M. performed dissections and imaging of post-implantation embryos. C.N.V. prepared RNA-seq libraries and performed RNA-seq analyses, and assisted with the preparation of SMCX/SMCY constructs. Y.M.N prepared ChIPSeq libraries. R.S.P. and P.M.G. performed ChIP-Seq analyses. All authors contributed to the writing and editing of the manuscript.

Availability of Data and Material. Sequencing data generated for this study have been submitted to the NCBI Gene Expression Omnibus (GEO; http://www.ncbi.nlm.nih.gov/geo/) under accession number GSE96740. All cell lines generated in this study are available upon request. 